Published in final edited form as:

Nat Biomed Eng. 2018 December ; 2(12): 930-941. doi:10.1038/s41551-018-0271-5.

\title{
A tissue-engineered scale model of the heart ventricle
}

\author{
Luke A. MacQueen ${ }^{\mathrm{a}}$, Sean P. Sheehya ${ }^{\mathrm{a}}$, Christophe O. Chantre ${ }^{\mathrm{a}}$, John F. Zimmermana, \\ Franceso S. Pasqualini ${ }^{a}$, Xujie Liue ${ }^{\mathrm{e}}$, Josue A. Goss ${ }^{\mathrm{a}}$, Patrick H. Campbella, Grant M. \\ Gonzalez ${ }^{\mathrm{a}}$, Sung-Jin Park ${ }^{\mathrm{a}}$, Andrew K. Capullia ${ }^{\mathrm{a}}$, John P. Ferrier ${ }^{\mathrm{a}}$, T. Fettah Kosar ${ }^{\mathrm{b}}$, L. \\ Mahadevan ${ }^{\mathrm{c}, \mathrm{d}}$, William T. Pue ${ }^{\mathrm{e}}$ Kevin Kit Parkera,e \\ ${ }^{a}$ Disease Biophysics Group, Wyss Institute for Biologically Inspired Engineering, John A. Paulson \\ School of Engineering and Applied Sciences, Harvard University, Cambridge, MA 02138, USA.
}

bWyss Institute for Biologically Inspired Engineering, Harvard Medical School, Boston, MA 02138, USA.

'John A. Paulson School of Engineering and Applied Sciences, Harvard University, Cambridge, MA 02138, USA.

dDepartment of Organismic and Evolutionary Biology, Department of Physics, Wyss Institute for Biologically Inspired Engineering, Kavli Institute for Nanobio Science and Technology, Harvard University, Cambridge, MA 02138S, USA.

eHarvard Stem Cell Institute, Harvard University, Cambridge, MA 02138 USA, Department of Cardiology, Boston Children's Hospital, Boston, MA 02115 USA

\section{Abstract}

\begin{abstract}
Laboratory studies of the heart use cell and tissue cultures to dissect heart function yet rely on animal models to measure pressure and volume dynamics. Here, we report tissue-engineered scale models of the human left ventricle, made of nanofibrous scaffolds that promote native-like anisotropic myocardial tissue genesis and chamber-level contractile function. Incorporating neonatal rat ventricular myocytes or cardiomyocytes derived from human induced pluripotent stem cells, the tissue-engineered ventricles have a diastolic chamber volume of $\sim 500 \mu \mathrm{L}$ (comparable to that of the native rat ventricle and approximately $1 / 250$ the size of the human ventricle), and ejection fractions and contractile work 50-250 times smaller and $10^{4}-10^{8}$ times smaller than the corresponding values for rodent and human ventricles, respectively. We also measured tissue coverage and alignment, calcium-transient propagation and pressure/volume loops in the presence or absence of test compounds. Moreover, we describe an instrumented bioreactor with ventricular-
\end{abstract}

\footnotetext{
Users may view, print, copy, and download text and data-mine the content in such documents, for the purposes of academic research, subject always to the full Conditions of use:http://www.nature.com/authors/editorial_policies/license.html\#terms

Corresponding Author: Kevin Kit Parker, Ph.D., Disease Biophysics Group, Wyss Institute for Biologically Inspired Engineering, John A. Paulson School of Engineering and Applied Sciences, Harvard University, 29 Oxford St, Pierce Hall 321, Cambridge MA 02138, kkparker@seas.harvard.edu.

Author contributions

L.A.M. and K.K.P. conceived the ideas and designed the experiments. L.A.M., S.P.S., C.O.C., J.F.Z., F.S.P., X.L., J.A.G., P.H.C., G.M.G., S.J.P., A.K.C., J.P.F., and T.F.K conducted the experiments and analyzed the data. L.M. derived the scaling laws. L.A.M., W.T.P., and K.K.P interpreted the data. L.A.M., S.P.S., and K.K.P. wrote the manuscript.

Competing interests

The authors declare no competing interests.
} 
assist capabilities, and provide a proof-of-concept disease model of structural arrhythmia. The model ventricles can be evaluated with the same assays used in animal models and in clinical settings.

\section{Introduction}

Laboratory models of the heart are used to gain a mechanistic understanding of heart function in health and disease, and to test the safety and efficacy of potential therapeutics. The heart is studied at multiple scales in vitro, from cellular assays to excised or engineered tissues and "organ-on-chip" microphysiological systems ${ }^{1}$ that recapitulate integrated aspects of specific pathological conditions. Functional readouts obtained using engineered heart tissues include contractile forces and electrophysiological measurements ${ }^{2}$, but direct comparison with natural ventricle pressure and volume dynamics requires three-dimensional (3D) contractile cardiac chambers. To obtain these measurements, animal models and isolated heart preparations are evaluated using pre-clinical and clinical measurement modalities such as catheterization ${ }^{3}$, echocardiography ${ }^{4}$, and magnetic resonance imaging ${ }^{5}$. Data obtained using these methods allow direct comparison to patient data, but differences in genetics, physiology and disease etiology limit the utility of animal models for developing therapeutic interventions ${ }^{6,7}$. These and other limitations associated with animal models, for example high maintenance costs and low experimental throughput, motivate the development of alternative in vitro cardiology assays based on human cells.

Human induced pluripotent stem cell-derived cardiomyocytes (hiPSC-CM) have emerged as a promising tool for in vitro cardiology, with the potential to eliminate interspecies and interpersonal variations through patient-specific derivation ${ }^{8}$. These cells are assembled into functional engineered heart tissues ${ }^{2,9,10}$, including muscular thin films ${ }^{11,12}$ or $3 \mathrm{D}$ muscle strips ${ }^{13-20}$, to model contractile pathophysiologies ${ }^{11}$, promote hiPSC-CM maturation ${ }^{9,14,15,21}$, and produce drug responses that are increasingly comparable to human patients ${ }^{16,18,22}$. However, the lack of in vitro models based on hiPSC-CM that accurately reproduce the architecture and functional output of the heart chambers ${ }^{9,10,23}$ is a significant limitation because tissue performance cannot be directly compared to animal or human heart performance, which are evaluated by measuring changes in chamber pressure and volume $^{24,25}$. Engineered cardiac organoid chambers comprised of cardiomyocytes embedded in isotropic hydrogels showed significant promise for obtaining intra-ventricular pressure measurements ${ }^{26-29}$ but the lack of a scaffold to guide cell assembly hampers the formation of organized tissues that recapitulate the laminar architecture ${ }^{30,31}$ of the native myocardium. Instead, we decided to use 3D scaffolds to provide anisotropic cues that guide the same laminar tissue formation we obtained on patterned monolayers ${ }^{32-35}$, and extend these design rules to tissues composed of multiple cell layers. Based on reports describing anisotropic cardiomyocyte assembly in fibrous scaffolds ${ }^{36-39}$, we reasoned that by taking design inspiration from the human myocardial tissue architecture and recreating it using a nanofiber production system, we could build 3D tissue-engineered ventricle scaffolds. Nanofibers provide biochemical and nano-topographical structural cues with sufficient fidelity to guide cell adhesion, orientation, shape, and assembly $y^{40,41}$. We therefore 
hypothesized that ventricle-shaped nanofibrous scaffolds would promote cardiomyocyte assembly into functional 3D tissue-engineered ventricle chambers.

To test if nanofibrous scaffolds are suitable for ventricle chamber tissue engineering, we first determined the minimal essential features of human ventricle structure that can be reliably recapitulated using nanofiber production systems. We fabricated ellipsoidal thin-walled chambers composed of a nanofibrous synthetic-natural polymer-protein blend and seeded them with neonatal rat ventricular myocytes (NRVM) or human induced pluripotent stem cell-derived cardiomyocytes (hiPSC-CM). Synchronous chamber-level contraction was observed for both cell types after 3-5 days of culture in the scaffolds. The resulting tissueengineered ventricle chambers were sutured to tubing or bioreactor components through which catheter sensors were introduced and stable contraction of both NRVM and hiPSC$\mathrm{CM}$ ventricles permitted time-dependent pressure and volume measurements. We then provide a proof-of-concept structural arrhythmia disease model by measuring spontaneous calcium activity of an engineered rat ventricle before and after inflicting geometrically controlled injuries. Our findings confirm that tissue-engineered model ventricle contraction can be monitored by catheter sensors, and suggest that model ventricles are suitable for cardiac arrhythmia studies. These results have implications for preclinical cardiology and regenerative medicine research where human organ models are sought to improve translation of therapeutic strategies.

\section{Model assumptions for tissue-engineered ventricle chambers}

To begin, we developed a model cardiac chamber based on tabulated structural and functional properties of the human left ventricle ${ }^{40}$. In the native heart, overlapping myocardial fibers are arranged into distinct laminae four to six myocytes thick and separated from adjacent laminae by an extracellular collagen network ${ }^{42}$. We reasoned that we could recapitulate the structure of model myocardial laminae within an ellipsoidal chamber geometry. Our overall strategy to produce a scale-model of the human left ventricle chamber for pressure-volume catheterization is depicted schematically in Figure 1a. We made the following assumptions from the outset: (i) We would build a scale-model of the human left ventricle chamber with diastolic chamber volume $\sim 500$ microliters $(\sim 2 \times$ rat, $\sim 1 / 250$ human); (ii) the ventricle wall would not be vascularized and its thickness limited to $\sim 0.1$ $\mathrm{mm}(\sim 1 / 10$ rat, $\sim 1 / 100$ human) to maintain high cell viability within the diffusion-limited environment; (iii) the ventricle scaffold material would be a well-known protein-polymer mixture to ensure production reproducibility using existing nanofiber systems; and (iv) cardiomyocyte alignment would be circumferential throughout the scaffold, representing a thin walled approximation of the helical alignment within the native ventricular wall.

\section{Ventricle scaffold}

Healthy ventricular musculature (myocardium) arises from precisely coordinated multiscale integration of physical forces transmitted between cells and the extra-cellular matrix (ECM), as well as between neighboring cells ${ }^{43}$. The myocardial ECM is fibrillar, anisotropic, and provides nanotopographical cues that guide cardiomyocyte alignment and assembly ${ }^{44}$, ultimately forming a helicoid structure that optimizes ejection fraction during ventricular 
contraction ${ }^{45}$. These features can be recapitulated using nanofibrous materials that are formed into cellular scaffolds with sufficient porosity to support cell infiltration and promote tissue morphogenesis ${ }^{40}$. We therefore developed a nanofiber ventricle chamber production strategy based on pull-spinning ${ }^{46}$ fibers on a rotating ellipsoidal collector (Fig. 1b, Supplementary Fig. S1, Supplementary Movie 1), which ensures roughly circumferential fiber alignment (Fig. 1c, Supplementary Movie 2). We selected polycaprolactone (PCL)/ gelatin nanofibers as our material of choice because they are biocompatible and biodegradable, they promote cell adhesion, provide sufficient structural integrity for ventricle culture and catheterization, and can be produced by a variety of nanofiber production systems that include electrospinning and force-spinning methods ${ }^{40}$. PCL provides structural stability for the scaffold's nanofibrous features during culture, while gelatin was chosen as an RGD-containing biopolymer derived from denatured collagen that improves scaffold binding to cells and biomolecules such as fibronectin ${ }^{47,48}$. We previously described anisotropic PCL/gelatin nanofiber production by pull spinning and their use to guide skeletal muscle tissue assembly ${ }^{46}$. Here, we used scanning electron microscopy (SEM) to confirm that PCL/gelatin nanofiber scaffolds were structurally comparable with decellularized human left ventricle tissue (Supplementary Fig. S2A). Gelatin incorporated into the nanofibers persisted for at least 4 days in aqueous solutions according to measurements made using X-ray photoelectron spectroscopy (Supplementary Fig. S2B). The tensile elastic modulus of our scaffolds was anisotropic, with values measured longitudinally (in the direction parallel to the fiber axis), $\mathrm{E}_{\mathrm{L}}=500 \pm 31 \mathrm{kPa}$, or transverse (in the direction perpendicular to the fiber axis), $\mathrm{E}_{\mathrm{T}}=74 \pm 15 \mathrm{kPa}$ (Supplementary Fig. S2C). These properties allowed us to fabricate ventricle scaffolds that were both structurally stable and permissive to chamber contraction by cardiomyocyte shortening.

Given that natural contraction rates of cultured cardiomyocytes are $~ 60-120$ bpm $(\sim 1-2$ $\mathrm{Hz})^{16,49}$, we designed chamber scaffolds that were mechanically permissive to contraction in the same range. To estimate the scaffold resonant bending frequency, $\omega_{B}$, we considered a simple balance between the kinetic and potential energy of a thin fluid-loaded spherical elastic shell of modulus, $E$, radius, $R$, fluid density, $\rho_{f}$, and thickness, $h^{50}$. The kinetic energy associated with the wall motion of amplitude, $A$, and frequency, $\omega_{B}$, scales as $\rho_{f} R^{3} A^{2} \omega_{B}^{2}$. The potential energy of deforming the shell is dominated by long wavelength bending deformations and scales as $E h^{3}\left(A / R^{2}\right)^{2} R^{2}$, where $E h^{3}$ is the bending stiffness, and the curvature scales as $A / R^{2}$. Balancing the two yields $E h^{3}\left(A^{2} / R^{2}\right) \sim \rho_{f} A^{2} R^{3} \omega_{B}^{2}$ so that $\omega_{B} \sim \sqrt{\left(E h^{3} / \rho_{f} R^{5}\right)}$. Using parameter values from our scaffolds $(R=5 \mathrm{~mm}, h=0.1 \mathrm{~mm}, 50$ $\mathrm{kPa}<E<500 \mathrm{kPa}$ ) gives bending resonant frequencies of $0.67 \mathrm{~Hz}<f_{B}<2 \mathrm{~Hz}$, where $f_{B}=$ $\omega_{B} / 2 \pi$, which match the contraction rates of cardiomyocytes. This suggests a natural design principal to engineer artificial ventricles as a function of the natural frequencies of cardiomyocyte contraction.

\section{Tissue-engineered ventricle chamber}

To build our model ventricle chambers, we seeded ventricle-shaped scaffolds with either neonatal rat ventricular myocytes (NRVM) or human induced pluripotent stem cell-derived 
cardiomyocytes (hiPSC-CM). Synchronous, coordinated ventricle contraction developed spontaneously after 3-5 days of culture (Supplementary Movies 3,4) and persisted for the duration of experimental procedures (Supplementary Movie 5), conducted 14 days after seeding. Calcium imaging, which has been used as a surrogate for the action potential ${ }^{51}$, revealed continuous excitation propagation on the ventricle surfaces, confirming electrical continuity throughout the ventricle constructs (Fig. 1d, Supplementary Movies 6,7). Mean calcium wavefront propagation velocity from apex to base was $9.33 \mathrm{~cm} / \mathrm{sec}$ or $5.2 \mathrm{~cm} / \mathrm{sec}$ for NRVM or hiPSC-CM ventricles, respectively. These values are comparable to those observed in tissues composed of immature cells where cell geometry, calcium handling, gap junction expression and spatial distribution, as well as other factors limit the conduction velocity when compared to mature tissues ${ }^{52,53}$. Immunostaining confirmed fiber-directed anisotropic cell alignment and infiltration within the chamber wall (Fig. 2, Supplementary Fig. S3, S4, Supplementary Movie 8), enabling us to quantify cell alignment using a metric known as the orientational order parameter (OOP) that ranges from zero (random organization) to one (perfect alignment) $)^{35,54}$. Both NRVM and hiPSC-CM ventricles showed high alignment based on OOP analysis of F-actin stains (Supplementary Fig. 3C), with OOP values of $0.84 \pm 0.02(\mathrm{NRVM})$ and $0.85 \pm 0.02$ (hiPSC-CM).

\section{Intra-ventricular pressure and volume measurements}

To determine whether our engineered ventricles exhibited in vivo like chamber contraction, we measured time-dependent intra-ventricular pressure and volume by catheterization (Fig. 3, Supplementary Fig. S5), an established method for heart chamber performance evaluation ${ }^{25}$. We sutured the engineered ventricles around tubing and inserted a pressure/ volume (PV) catheter into the chamber midway from base to apex (Supplementary Fig. S5A). We performed catheter calibrations (Supplementary Fig. S5) and acquired 2,000 data points per second (Supplementary Fig. S6). Exported data was processed for time and frequency-domain analysis, which are commonly used to quantitatively assess cardiovascular function ${ }^{55,56}$ (Supplementary Fig. S7). As an initial validation of our experimental system, we investigated ventricle contractile responses to gross alterations in the composition of the extracellular bath solution. Whereas regular beat frequencies were observed in calcium-containing M199 or Tyrode's solution, exposure to calcium-free PBS led to broad beat rate distributions (Supplementary Fig. S7B). Furthermore, we observed an expected reduction in beat rate, as judged by a shift in the frequency spectrum, as bath temperature was decreased from physiological $37^{\circ} \mathrm{C}$ to less than $\sim 30^{\circ} \mathrm{C}$ (Supplementary Fig. S7C). These results demonstrate that tissue-engineered model ventricles can be functionally interrogated and myocardial performance assessed using catheter-based pressure-volume measuring systems. To visualize the relationship between pressure and volume change during cardiac contraction cycles, we plotted PV loops for both rat (NRVM) and human (hiPSC-CM) ventricles from PV catheter measurements (Fig. 3b). Measured differences in chamber pressure were $\sim 50 \mu \mathrm{mHg}$ (rat or human) and the volume change was $\sim 5 \mu \mathrm{L}$ (rat) or $1 \mu \mathrm{L}$ (human). Thus, ejection fractions were $\sim 1 \%$ (rat) or $\sim 0.2 \%$ (human) and stroke work was $\mathrm{W}_{\mathrm{S}} \sim 0.25 \mathrm{mmHg} \times \mu \mathrm{L}$ (rat) or $\mathrm{W}_{\mathrm{S}} \sim 0.05 \mathrm{mmHg} \times \mu \mathrm{L}$ (human).

A fundamental aspect of myocardial function is the response to adrenergic agonists, which has been studied extensively in both rodents and humans, making $\beta$-adrenergic response a 
good test of the ability of our engineered ventricles to recapitulate normal cardiac pharmacology 57,58 . To assess the chronotropic response of our engineered ventricles to a $\beta$ adrenergic receptor agonist, we subjected them to concentrations of isoproterenol ranging from $0.1 \mathrm{nM}$ to $0.1 \mathrm{mM}$. Pressure/volume loops measured before and after isoproterenol exposure showed an isoproterenol-induced reduction in stroke work, concomitant with an increase in beat frequency (Fig. 3b,c). Continuous intra-ventricular pressure and volume measurements enabled frequency-domain analysis of the chronotropic response of either NRVM or hiPSC-CM ventricles (Supplementary Fig. S7D). The spontaneous beat rate of NRVM ventricles $(\sim 130 \pm 15 \mathrm{bpm})$ was higher than hiPSC-CM ventricles $(\sim 85 \pm 15 \mathrm{bpm})$, and both increased by $\sim 40 \%$ following exposure to $10^{-4} \mathrm{M}$ isoproterenol. Ventricle beat rates showed a positive chronotropic response over the concentration range in both the NRVM and hiPSC-CM ventricles (Fig. 3c), as expected for healthy NRVM ${ }^{59}$, hiPSC-CM ${ }^{60}$, engineered hiPSC-CM cardiac tissues ${ }^{16}$, and human patients ${ }^{61}$.

\section{Bioreactor for modular assembly of ventricles and valves}

Laboratory models of biological tissues often benefit from culture in bioreactors or microfluidic technologies where fluid exchange can be automated and in situ measurements obtained. To support tissue-engineered ventricle culture, we built a self-contained instrumented heart bioreactor (HBR; Fig. 4, Supplementary Figs. S8,S9, Supplementary Movie 9). The HBR includes both intra- and extra- ventricular flow loops, separate chambers for optional valve inserts, and additional access ports for catheters (Fig. 4a, Supplementary Fig. S8). Cyclic pressure applied to the extra-ventricular loop drives intraventricular fluid flow via assisted ventricle contraction, and unidirectional flow can be achieved using commercially available valves or with custom valve inserts in the HBR. We demonstrated assisted pumping in the absence of drugs by controlling pressure delivered to the external (assist) channel using a pressure-driven programmable pump and cast-molded silicone tricuspid valves to direct flow (Fig. 4a, Supplementary Fig. S9). In this configuration, cultured ventricles can be exposed to externally-controlled physiological or pathological pressure variations and PV loops acquired with or without valves (Fig. 4b), emphasizing the potential to study heart failure where differential effects of various organ sub-structures are measured. Lastly, to highlight the translational nature of this technology, we monitored ventricle contraction by ultrasound through removable elastomeric windows in the HBR (Fig. 4c, Supplementary Fig. S10, Supplementary Movies 10,11). Using small animal echocardiography equipment, we recorded both unassisted natural spontaneous ventricle contraction (Fig. 4c, left, and Supplementary Movie 10) and HBR-assisted contraction (Fig. 4c, right, and Supplementary Movie 11). Echocardiography performed in the absence of extra-ventricular tissues can improve image clarity compared with in vivo studies, underscoring the advantage of organ modularity in our HBR.

\section{Structural arrhythmia disease model}

In myocardial tissues, anatomical structures can introduce heterogeneities of impulse conduction that lead to ventricular tachycardia, including spiral waves anchored to anatomical defects ${ }^{33,62}$. Here, we show a proof-of-concept structural arrhythmia disease model by measuring spontaneous calcium activity of an engineered rat (NRVM) ventricle 
before and after inflicting geometrically controlled (1-mm diameter) injuries (Fig. 5). Plane wave propagation was observed prior to injury (Fig. 5a, top), whereas injured ventricles generated spiral waves that were pinned to the controlled anatomical defects (Fig. 5a, bottom panels, and Supplementary Movie 12). Calcium fluorescence intensity measured near the rotor poles of a single-hole injury model showed consistent phase difference and a rotation rate of $\sim 5 \mathrm{~Hz}$ (Fig. 5b, Supplementary Figure S12, and Supplementary Movie 13). The twohole injury generated counter-propagating spiral waves that merged within the region between holes (Fig. 5a, third row). In all cases, spontaneous calcium activity showed stable excitation patterns with plane waves (healthy) or pinned spiral waves (injured) repeating at a near-constant rate (between $4 \mathrm{~Hz}$ and $5 \mathrm{~Hz}$ ) for the duration of experiments ( 2 hours). These results suggest the feasibility of using tissue engineered heart chambers to study arrhythmogenic heart diseases.

\section{Discussion}

Preclinical cardiology and regenerative medicine research will benefit from an expanding set of in vitro models where human heart structure and function are examined at multiple scales $2,9,10,63$. Here, we took inspiration from the myocardial ECM and used nanofibers to extend to $3 \mathrm{D}$ tissue engineered cardiac chambers the same engineering design principles that led to increasingly functional laminar cardiac tissues ${ }^{32,34,64}$. For near-future design iterations that concentrate on myocardial engineering, we suggest several steps that may precede the addition of non-cardiomyocytes and ventricle vascularization ${ }^{65}$. These "obstacles and opportunities" facing bioartificial hearts ${ }^{66}$ include improvements to scaffolds, cardiomyocytes, and culture protocols, briefly outlined below.

Scaffold engineering should account for the fact that the myocardium is a composite in which the dominant stiffness shifts from myocytes to the ECM with increasing strain ${ }^{67}$, emphasizing the ECM's role maintaining structural integrity. In our scaffold, gelatin was used to enhance cell and biomolecule adhesion during cell seeding, and the polymer backbone (PCL) provided structural features (microporosity, nanotopography, anisotropy) that resisted degradation during culture. These features provided sufficient structural integrity for ventricle culture and they guided cardiomyocyte assembly but alternative nanofibrous materials used for cardiac tissue-engineering ${ }^{40}$ may improve scaffold customization. For example, the tensile elastic modulus of our scaffold in the direction of fiber alignment $\left(\mathrm{E}_{\mathrm{L}} \sim 500 \mathrm{kPa}\right)$ was higher than healthy ventricular $\mathrm{ECM}(\mathrm{E} \sim 350 \mathrm{kPa})^{68}$ and may have limited cardiomyocyte shortening during contraction. The balance between structural support during culture, scaffold degradation rate, and ventricle mechanics should be refined. Alternative biodegradable polymers or bioprotein mixtures reflecting agedependent composition of natural myocardial $\mathrm{ECM}^{40}$ may be used to improve scaffold elasticity, recapitulate specific disease or development phases, or promote scaffold replacement with cell-secreted ECM. Biomolecular nanofibers can be produced using precipitation systems ${ }^{69}$ for direct use as scaffolds or dispersed in printable "inks", where ink and print conditions influence printed nanofiber orientation ${ }^{70}$. Our work suggests that including a nanofibrous component to printable inks would be advantageous for directing cardiomyocyte shape and assembly in printed scaffolds or tissues. Alternative chamber shapes, anatomical features, and scaffold fiber alignments may be used to study their effects 
on cardiomyocyte assembly and electromechanical integration. In all cases, design rules for contractile 3D chambers should account for chamber contraction as a function of chamber size, shape, and elastic modulus. Our calculations of resonant bending frequencies suggests a natural design principal to engineer cardiac chambers as a function of their intended beat rates.

Cardiomyocyte spontaneous beat rates in our model ventricles were similar to other in vitro assays based on $\mathrm{NRVM}^{49}$ or hiPSC-CM ${ }^{16}$, showing moderate positive chronotropy ( 40\% beat rate $)^{10,18}$. We expected to observe variance between ventricles, both in terms of beat rates and their response to isoproterenol, given the heterogeneous pace-making phenotypes within the cardiomyocyte populations ${ }^{10}$. Our observation of isoproterenol-induced stroke work reduction (Fig. 3b) is consistent with negative force-frequency relationships observed in tissues based on immature cardiomyocytes, including $\mathrm{NRVM}^{71}$ and hiPSC-CM ${ }^{72}$. The moderate positive chronotropy and reduction of stroke work observed at high isoproterenol doses are characteristic of immature tissues exhibiting poorly developed calcium handling properties $^{8}$, suggesting that maturation protocols $\mathrm{s}^{14,21,22,73}$ may be required to obtain positive force-frequency relationships, as observed in healthy adult human ventricular myocardium ${ }^{74}$.

Critical to the advance of tissue-engineered ventricles will be the development of in situ stem cell proliferation, differentiation, and maturation protocols. The cell density in our ventricles, estimated by counting immunostained (DAPI) NRVM ventricle surfaces and cross-sections (Supplementary Fig. S3), was $\sim 90,000$ cells $/ \mathrm{mm}^{3}$ for a total cell number $\sim 2.3$ $\times 10^{6}$ in a $26 \mathrm{~mm}^{3}$ scaffold volume, which is approximately 10 -fold less than in 21-day-old or 3-month-old rat hearts ${ }^{75}$. The EF of our tissue-engineered rat ventricles was $\sim 1 \%(1 / 50$ EF of healthy rats), suggesting that discontinuities in cardiomyocyte coverage limited tissueengineered ventricle contractile strength. Novel protocols that proliferate stem cells to confluence within the scaffolds may overcome the coverage limitation, provided that in situ differentiation and maturation can be efficiently achieved following the proliferation phase. Preliminary work in our laboratories suggest that we can achieve extended culture periods for NRVM (Supplementary Fig. S12, Supplementary Movies S14, 15) and hiPSC-CM (Supplementary Figs. S13, S14) in our nanofibrous scaffolds. For example, proliferative hiPSC (line PGP1-iPSC) seeded in our scaffolds at a density of $10^{5} \mathrm{cells} / \mathrm{cm}^{2}$ and cultured according to previously described protocols ${ }^{11}$ achieved confluence within 4 days of culture (Supplementary Figure S13A, Day 0). When cultured in differentiation media, the resulting hiPSC-CM showed spontaneous contraction and early stages of sarcomeric assembly within 8-12 days of culture (Supplementary Figure S13A, Day 12), and anisotropic sarcomeric assembly with early stages of z-disk alignment after 1 month in culture (Supplementary Figure S13A, Day 30). We also cultured a non-proliferative hiPSC-CM line (Cor.4U) in model ventricle scaffolds for 6 months, demonstrating that tissue anisotropy was preserved (Supplementary Figure S14A) and stable pressure-volume loops could be recorded (Supplementary Figure S14 B). These preliminary results suggest that our scaffolds support functional myocardial tissue production using relatively small numbers of seeded hiPSC that can subsequently be cultured for extended time periods. In these cases, tissue maturation may benefit from bioreactor culture where tissues are exposed to physiological pressures and electromechanical stimulation. Similarly, patho-physiological studies using healthy and 
diseased human cells that may respond positively to ventricular assist should also be conducted. These future experiments, using systems like ours to evaluate hiPSC-CM tissue formation and electromechanical integration within biosynthetic scaffolds, will inform regenerative medicine practices that aim to restore myocardial function ${ }^{63,76,77}$.

A direct comparison of engineered ventricle chamber contractile performance with those of ex-vivo animal studies is challenging. For example, our thin wall chambers powered by immature cardiomyocytes generated small differences in chamber pressure $(\Delta \mathrm{P} \sim 50 \mu \mathrm{mHg})$ and chamber volume $(\Delta \mathrm{V} \sim 1-5 \mu \mathrm{L})$, equivalent to ejection fractions, $\mathrm{EF} \sim 0.2-1 \%$, which are smaller than expected for healthy mammalian ventricles by factors of roughly $50-250$. Similarly, the contractile work performed by our ventricles $\left(\mathrm{W}_{\mathrm{S}} \sim 0.05-0.25 \mathrm{mmHg} \times \mu \mathrm{L}\right.$, or $\mathrm{W}_{\mathrm{S}} \sim 7-35 \mathrm{~nJ}$ ) was less than mouse, rat, or human left ventricles by factors of $\sim 10^{4}, 10^{5}$, and $10^{8}$, respectively ${ }^{24}$. Contractile work in our ventricle models was measured using conductance catheters and was smaller than reported for cardiac organoid chambers based on $\mathrm{NRVM}^{26}$ or pluripotent hES2 human embryonic stem cell-derived cardiomyocytes ${ }^{29}$, where volume changes were indirectly assessed from video recordings. Both types of model cardiac chambers generated similarly shaped elliptical PV-loops (Fig. 3b, Supplementary Fig. S14), which are expected from ventricles contracting in the absence of valves and show similarities to PV loops obtained from patients with severe valvular insufficiencies ${ }^{78}$. Our use of conductance catheters was advantageous for direct volume readouts but electrical pacing during catheterization was not recommended by the catheter manufacturer and therefore not performed in these studies. Thus, the positive inotropic effects of isoproterenol observed by others during electrical pacing ${ }^{29}$ was not observed by us when isoproterenol was administered to spontaneously beating model ventricles (Fig. 3b, right panels). One solution to this challenge may be the use of optogenetics for optical pacing ${ }^{79}$. Scaling of chamber contractile strength with cardiomyocyte number is expected and it is encouraging to note that contractile strength increased in our preliminary extended culture period experiments (Supplementary Fig. S14). Lastly, our structural arrhythmia disease model reaffirmed the importance of building native-like anisotropic tissues that are important for both normal propagation and arrhythmogenesis ${ }^{33}$. Precise anatomical defects applied to our tissue engineered model ventricles generated stable pinned rotors and spiral waves (Fig. 5, Supplementary Fig. 11, Supplementary Movies S12, S13) that have so-far not been demonstrated in engineered cardiac chambers.

In summary, our results demonstrate the feasibility of engineering functional scale models of the heart chambers, where tissue assembly is guided by a nanofibrous culture substrate, with implications for multiscale in vitro cardiology assays and regenerative medicine research. We used primary rat cardiomyocytes and human stem cell-derived cardiomyocytes because rat is the current industry standard for the study of heart disease in vitro and in vivo, yet the use of human models will be key for personalized medicine. In this respect, we believe it is important to include use of pre-/clinical grade measurement technologies to provide a more seamless transition between in vitro and in vivo models. This will extend patient-specific in vitro assays from individual cells to complete organ models, and our design rules provide a path toward engineering heart chambers with functional performance increasingly comparable to native organs. Taken together, the capability to tissue-engineer functional model ventricles, and the modular bioreactor technologies designed for in situ 
instrumentation and functional assessment, provide a suitable system for in vitro cardiology studies that increasingly translate to clinical outcomes.

\section{Methods}

\section{Scaffold fabrication}

Polymer solutions. Ventricle scaffold material precursors were polycaprolactone (PCL; Sigma Aldrich 440744) and gelatin Type A (Sigma Aldrich G2500) dissolved in a solvent, 1,1,1,3,3,3-Hexafluoro-2-propanol (HFIP; Oakwood Chemical 003409). All materials were used as received without further modifications. A mixture of 75\% PCL and 25\% gelatin was dissolved at $6 \%$ weight/volume in HFIP and stirred for at least $12 \mathrm{hr}$ to ensure homogeneous mixing. Ventricle scaffold fabrication. Ventricle scaffolds were produced by pull spinning ${ }^{46}$ (PS; Fig. 1b, Supplementary Fig. S1A) PCL/gelatin nanofibers onto ellipsoidal collection mandrels (half-ellipsoid with radii: $\mathrm{a}=\mathrm{b}=4.5 \mathrm{~mm}, \mathrm{c}=9 \mathrm{~mm}$; Supplementary Fig. S1B). Pull spinning incorporates a high-speed (up to 45,000 RPM) rotating bristle that dips into a fixed, continuous polymer source and pulls the polymer column into an anisotropic network of non-woven nanofibers. The solution was injected through a needle (18G flat tip; BD Biosciences) at a rate of $0.2 \mathrm{~mL} / \mathrm{min}$ for a total duration of $5 \mathrm{~min}$. Bristle rotation rate was 30,000 RPM. Ventricle mandrels were connected to a DeWALT DC 720 1/2" cordless drill driver rotation operating at $300 \mathrm{RPM}$ and the mandrels were located $20 \mathrm{~cm}$ from the polymer source. All PS fabrication was performed within a chemical fume hood and the relative humidity was between 10-20\%. Scaffolds were stored in de-ionized water for 48 hours and sterilized by overnight exposure to UV radiation within a tissue culture hood prior to use. Overnight exposure to UV was sufficient to prevent bacterial contamination but future work with thicker scaffolds may benefit from more thorough sterilization procedures.

\section{Scaffold structural and biochemical analysis}

$X$-ray Microcomputed Tomography $(\boldsymbol{\mu C T})$. $\mu \mathrm{CT}$ was performed at Harvard University's Center for Nanoscale Science (CNS). We used an X-Tek HMXST225 system (Nikon Metrology, Inc.) equipped with a $225 \mathrm{kV}$ microfocus X-ray source with $3 \mu \mathrm{m}$ focal spot size. We used an aluminum target and $115 \mathrm{kV}$ accelerating voltage. Image acquisition and reconstruction was performed using the following software suites: InspectX (X-ray imaging and CT acquisition), CT Pro 3D (volume reconstruction), VG Studio MAX 2.2 (3D volume visualization, rendering and analysis), and Amira (3D volume visualization, rendering and analysis). Scanning electron microscopy. All SEM imaging was done using a field emitting electron microscope (FESEM Ultra Plus, Zeiss) at a voltage of $15 \mathrm{kV}$ to image scaffold and tissue fiber alignment. Prior to imaging, all samples were sputter coated with $5 \mathrm{~nm}$ of Platinum/Palladium (Pt/Pd) using a Quorum Sputter Coater (EMS 300T D, Quorum Technologies) to reduce charge accumulation and tissue decomposition during imaging. Decellularized human tissue was prepared using a previously published SDS profusion protocol ${ }^{80}$ and dehydrated in serial ethanol washes. Dehydrated tissues were then dried using a SAMDRI critical point drier (931 Series SAMDRI, Tousimis) prior to sputter coating to ensure complete removal of interstitial fluids. Mechanical Testing. To measure elastic modulus, we produced sheets of nanofibrous material (Supplementary Figs. S1, S2), collected on flat glass coverslips, using the same pull-spinning conditions as for ventricle 
chamber production. Square samples were laser-cut $(10 \times 10 \mathrm{~mm})$ from these sheets and loaded onto $5 \times 5 \mathrm{~mm}$ mounting tines for biaxial tensile testing ( $2.5 \mathrm{~N}$ load cells; Biotester, CellScale, Canada). A pre-stress of $5 \mathrm{mN}$ was applied before running four biaxial preconditioning cycles at $5 \%$ strain rate to $20 \%$ strain. Using the original dimensions of the nanofibrous samples, the strain-strain curve was then calculated (same strain rate and strain were used as preconditioning). To replicate in vitro conditions, tensile measurements were performed in PBS at $37{ }^{\circ} \mathrm{C}$. X-ray photoelectron spectrometry. A K-Alpha X-ray photoelectron spectrometer and Advantage software (K-Alpha XPS, Thermo Scientific) was used to evaluate fresh-spun and wetted scaffold composition in time. $5 \times 5 \mathrm{~mm}$ pieces of $75 / 25$ PCL/Gelatin scaffold were wetted in 1L of ultra-pure water and stored in an incubator at $37^{\circ} \mathrm{C}$ for up to 1 week. Fresh spun and wetted samples removed from the water bath daily were dried for $12 \mathrm{hr}$ under vacuum and their composition evaluated using the XPS system ( $\mathrm{n}=3$ scaffold pieces and XPS measurements per time point, 0-7 days). Briefly, each sample was etched for $30 \mathrm{~s}$ at $500 \mathrm{eV}$ to remove surface debris and was survey scanned over a 400 square micron spot size. Gelatin content was estimated based upon the measured presence of nitrogen in the sample and the amount of solvent (HFIP) was estimated based upon the measured presence of fluorine, each normalized to the element's percentage within their respective molecule. Fourier Transform Infrared Spectroscopy (FT-IR). We used a Bruker FT-IR Microscope (Lumos, Bruker, Billerica, MA) in attenuated total reflection (ATR) mode to measure the infrared spectra of the nanofibers. Data plotting was conducted with custom software written in MATLAB (MathWorks, Natick, MA).

\section{Experimental animals}

Neonatal rat ventricular myocytes (NRVM) were isolated from 2-day old neonatal CRL: CD (SD) rats, and left ventricle histological preparations were obtained from adult female CRL: CD (SD) rats. All procedures were approved by the Harvard Animal Care and Use Committee and all research personnel handling animals were appropriately qualified and trained by Harvard's Office of Animal Resources, under the direction of the Attending Veterinarian. Pups were euthanized using a method consistent with the recommendations of the 2013 American Veterinary Medical Association (AVMA) Guidelines on Euthanasia for rodents. Care and Use of the rats used in this study comply with the "US Government Principles for the Utilization and Care of Vertebrate Animals Used in Testing, Research, and Training", the Guide for the Care and Use of Laboratory Animals, and the Animal Welfare Act/Regulations. Harvard University, Faculty of Arts and Sciences ("FAS") maintains an Institutional Animal Care and Use Committee (IACUC) as required by the Public Health Service (PHS) Policy on Humane Care and Use of Laboratory Animals. All animal protocols must be approved by the IACUC before animals can be ordered.

\section{Cell and Tissue Culture}

Neonatal rat ventricular myocytes (NRVM). NRVM were isolated from 2-day old neonatal neonatal CRL: CD (Sprague-Dawley, SD) using published methods ${ }^{64}$. All procedures were approved by the Harvard Animal Care and Use Committee. Cells were seeded at a density of 3 million cells per ventricle, following procedures described below. Standard culture media were used (M199 culture medium supplemented with 0.1 mM MEM nonessential amino acids, $10 \%$ heat-inactivated FBS, 10mM HEPES, $3.5 \mathrm{~g} / \mathrm{L}$ glucose, $2 \mathrm{mM} \mathrm{L-glutamine,} 2$ 
$\mathrm{mg} / \mathrm{L}$ vitamin B-12, and $50 \mathrm{U} / \mathrm{ml}$ penicillin). Samples were incubated under standard conditions at $37{ }^{\circ} \mathrm{C}$ and $5 \% \mathrm{CO}$. At $48 \mathrm{~h}$ post seeding the media was exchanged with maintenance media (M199 media supplemented as above but with 2\% FBS) and was exchanged again every $48 \mathrm{~h}$ until use. Human induced pluripotent stem cell-derived cardiomyocytes (hiPSC-CM). hiPSC-CMs were acquired commercially (Lot numbers CB169CL_V1_1M, CB301_CL_v1_1M, CB319CL_V1_1M, CB324CL_V1_1M, CB331CL_V1_4M; Axiogenesis, Cologne, Germany) and cultured according to manufacturer's instructions with slight modifications. Briefly, for each cryovial containing 1 million viable hiPSC-CMs, 3 wells of a 6 well tissue culture plate were coated with 0.01 $\mu \mathrm{g} / \mathrm{mL}$ fibronectin (FN) (BD Biosciences, Bedford, MA) for 4 hours in a $37^{\circ} \mathrm{C}$ incubator. According to the manufacturer, Cor.4U cells show typical ventricular-, atrial- and nodal-like action potentials, demonstrating the different Cor.4U subtypes. Cor.4U comprises $60 \%$ ventricular cells. Cryovials of Cor.4U hiPSC-CMs were quickly thawed in a $37^{\circ} \mathrm{C}$ water bath, re-suspended in $10 \mathrm{~mL}$ of complete culture medium provided by the manufacturer supplemented with $5 \mu \mathrm{L}$ of $10 \mathrm{mg} / \mathrm{mL}$ puromycin, and cultured in FN-coated tissue culture plates at $37^{\circ} \mathrm{C}$ and $5 \% \mathrm{CO}_{2}$ for 48 hours to eliminate un-differentiated stem cells from the culture. After 48 hours, cells were dissociated with $0.25 \%$ trypsin and seeded onto ventricular scaffolds. Human induced pluripotent stem cell-derived cardiomyocyte (hiPSCCM) line PGP1-iPSC. To demonstrate in situ hiPSC proliferation and hiPSC-CM differentiation in our nanofibrous scaffolds, we performed preliminary experiments using the human iPSC line PGP1-iPSC, which we have used previously for monolayer muscle thin film assays ${ }^{11}$. hiPSC were seeded on the surface of nanofibrous sheets at a density of $10^{5}$ cells $/ \mathrm{cm}^{2}$ and proliferated to confluence within four days of culture in $\mathrm{mTeSR}{ }^{\mathrm{TM}}$ culture medium (Supplementary Figure S13 A, left panels). hiPSC-CM differentiation was then induced by changing media from mTeSR ${ }^{\mathrm{TM}}$ to RPMI/1640+B27-minus insulin with $8 \mathrm{uM}$ CHIR 99021, incubating for 2 days, and subsequently changing media to RPMI/1640+B27minus insulin with 5 uM IWR-endo-1, and incubated for another 2 days. We then maintained the culture with fresh RPMI/1640+B27 media every other day. Tissue contraction was observed following 8-12 days of differentiation, concomitant with early stages of sarcomeric expression and assembly (Supplementary Figure S13 A, middle panels). Contractile synchrony improved during extended culture periods, concomitant with increased sarcomeric assembly and myocardial tissue anisotropy (Supplementary Figure S13 A, right panels). Immunofluorescence staining confirmed 3D infiltration of hiPSC-CM within our nanofibrous scaffolds, including early stages of z-disk alignment (Supplementary Figure S13 B). Ventricle Seeding. We sterilized ventricle scaffolds by exposure to ultraviolet radiation in a biosafety hood overnight. The following morning, we incubated the ventricle scaffolds with $100 \mu \mathrm{g} / \mathrm{mL}$ fibronectin (Human natural fibronectin, BD Biosciences) in PBS for 90 minutes, then transferred cells to the scaffold at high density $(0.5 \mathrm{~mL}$ full media containing $3 \mathrm{M}$ cells) and incubated $\left(37^{\circ} \mathrm{C}, 5 \% \mathrm{CO}_{2}\right)$ for 90 minutes. $2 \mathrm{~mL}$ of full media was then added to each well and incubated overnight. Ventricles were then transferred larger wells, each well containing $5 \mathrm{~mL}$ full media and one ventricle, with media refreshed every 48 hours until use. 


\section{Histochemical staining}

Cardiomyocyte infiltration and orientation within tissue-engineered ventricles was visualized by fluorescent staining of actin and sarcomeres. We stained for F-actin fibers and sarcomeric a-actinin as previously described ${ }^{64}$. Briefly, washed samples were fixed in $4 \%$ paraformaldehyde for 20 minutes. To prepare ventricle cross-sections, we first cast gelatin molds of the ventricular volume by pouring dissolved gelatin $(20 \% \mathrm{w} / \mathrm{v})$ into ventricleshaped molds, followed by cooling and removal of the solid gelatin from the mold. We then pulled fixed ventricles over the gelatin mold to ensure that thin-walled ventricles maintained an ellipsoidal shape during cryopreservation. Ventricles (with gelatin interior) were stored in PBS $+30 \%$ sucrose solution overnight at $4^{\circ} \mathrm{C}$, transferred to $50 \%$ sucrose $/ 50 \%$ OCT for 24 $\mathrm{h}$ at $4{ }^{\circ} \mathrm{C}$. They were then transferred to cryosectioning containers in $100 \%$ OCT and stored at $4{ }^{\circ} \mathrm{C}$ for $48 \mathrm{~h}$. Samples were frozen by partial immersion in 2-methylbutane which was, itself, partially immersed in liquid nitrogen. Frozen ventricles were stored at $-80{ }^{\circ} \mathrm{C}$ until cryosectioning by microtome (Leica). We obtained $30 \mu \mathrm{m}$-thick cross-sections that were transferred to glass microscope slides (Superfrost microscope slides, Sigma) and maintained at room temperature for $2 \mathrm{~h}$ prior to storage at $-80{ }^{\circ} \mathrm{C}$ until staining. Staining and imaging. Ventricle surfaces or cross-sections were permeabilized in $0.5 \%$ Triton-X100 for 20 minutes in PBS at $37{ }^{\circ} \mathrm{C}$, followed by 2-h incubation with 1:200 dilutions of mouse anti-sarcomeric a-actinin monoclonal primary antibody (Sigma-Aldrich, clone EA-53, catalog number A7811-11UL). Samples were then washed and concurrently incubated with 1:200 dilutions of DAPI (Sigma-Aldrich), phalloidin conjugated to Alexa-Fluor 488 (In vitrogen, Carlsbad, CA 92008, USA) and goat anti-mouse secondary antibody conjugated to tetramethylrhodamine for $2 \mathrm{~h}$ at room temperature. Imaging was performed using a Zeiss LSM 5 LIVE confocal microscope with a Plan-Neofluar 40x/1.3 oil objective. For long range myocardium tissues, samples were imaged using an Olympus IX83 microscope with an attached Andor spinning disk confocal system, on LUCPlanFLNPh 20x and 40x objectives, and were recorded on a Hamamatsu Orca Flash 4.0 C11440 at 16bit depth. Zstacks were collected over the height of a single myocardium wall, and then sections were spliced together using a standard deviation projection followed by a pairwise stitching algorithm in NIH ImageJ. We quantified cell alignment using a metric known as the orientational order parameter (OOP) that ranges from zero (random organization) to one (perfect alignment $)^{35,54}$, applied to immunostained F-actin.

\section{Optical mapping experiments}

Calcium propagation was monitored using a modified tandem-lens macroscope (Scimedia, Costa Mesa, CA) equipped with a high speed camera (MiCAM Ultima, Scimedia, Costa Mesa, CA), a plan APO 0.63× objective, a collimator (Lumencor, Beaverton, OR) and a 200 $\mathrm{mW}$ Mercury lamp (X-Cite exacte, Lumen Dynamics, Canada). After 2 weeks culture in vitro, ventricles were incubated with $2 \mu \mathrm{M}$ Rhod-2 (Invitrogen, Carlsbad, CA) for $30 \mathrm{~min}$ at $37{ }^{\circ} \mathrm{C}$, rinsed, and incubated in dye free media for an additional $15 \mathrm{mins}$ at $37^{\circ} \mathrm{C}$ prior to recording. The ventricles were then rinsed with Tyrode's buffer. Recordings were acquired at a frame rate of $200 \mathrm{~Hz}$. Electrical field and point simulation was applied using two platinum electrodes (Sigma-Aldrich, St. Louis, MO) with $20 \mathrm{~mm}$ and $1 \mathrm{~mm}$ spacing, respectively. The point stimulator was located at $0.5-1 \mathrm{~mm}$ from the apex of the ventricle with a motorized 
xy-micromanipulator (Zaber Technologies Inc., Vancouver, British Columbia, Canada). Electrical pulses were generated with $12 \mathrm{~V}$ amplitude and $10 \mathrm{~ms}$ duration using a pulse generator (MyoPacer Cell Stimulator, IonOptix, Milton, MA). Pacing frequency was $1 \mathrm{~Hz}$ for NRVM ventricles and $3 \mathrm{~Hz}$ for hiPSC-CM ventricles ( $3 \mathrm{~Hz}$ pacing was required in this case because lower pacing frequencies were obfuscated by spontaneous activity). Postprocessing of data was conducted with custom software written in MATLAB (MathWorks, Natick, MA). A spatial filter with $3 \times 3$ pixels was applied to improve the signal-noise ratio. Activation time of point-stimulated ventricle was calculated as the average time to maximum upstroke slope of pulses when continuously at $1 \mathrm{~Hz}$ pacing during in a $5 \mathrm{sec}$ recording window. For structural arrhythmia disease modelling, we built four NRVM model ventricles (two healthy and two pre-injured with circular holes of $1 \mathrm{~mm}$ diameter), and spontaneous calcium activity was monitored in all cases. Calcium mapping of pre-injured ventricles was done on day 11 but tissue had grown into the injury site, preventing stable rotor pinning. We repeated calcium mapping experiments on day 12 using the two uninjured ventricles. Both showed evidence of spontaneously generated calcium plane wave propagation but one had greater homogeneity; this favorable sample is reported in our revised manuscript. For calcium imaging, tissue engineered ventricles were placed in a temperature-controlled petri dish and imaged from above. We first imaged an uninjured NRVM ventricle with $5 \mathrm{msec}$ exposure windows and a $2.5 \mathrm{sec}$ recording window. We then used a 1-mm diameter biopsy punch to perforate the ventricle wall and repeated calcium imaging. Lastly, we perforated the ventricle with a second 1-mm diameter hole spaced $5 \mathrm{~mm}$ from the first hole and repeated calcium imaging. Analysis was done using MiCAM imaging software (BV_Ana, SciMedia) and custom-software derived from a publicly available MATLAB-based optical mapping analysis package (Rhythm2014b) ${ }^{81}$. This ventricle was then fixed and stained to evaluate cell coverage by confocal immunofluorescence imaging (Supplementary Figure S4). Although our model ventricles were placed in temperature-controlled petri dishes, with a roughly $2 \mathrm{D}$ geometry during calcium imaging, we showed that ventricles could be suspended in our bioreactor chambers (Fig. 4), suggesting future compatibility with isolated heart perfusion systems currently used for small animal cardiology.

\section{Pressure-Volume Measurements}

For pressure-volume experiments, we built a total of 20 rat (NRVM) and 10 human (Cor.4U) model ventricles. Of these, 12 rat and 6 human ventricles were selected for catheterization based on the synchrony and amplitude of contraction observed in vitro by eye and microscope. We report data for 8 rat and 4 human ventricles, for which pressure or volume recordings were discernible at each of 7 isoproterenol doses $(0.1 \mathrm{nM}$ to $0.1 \mathrm{mM}$; total experiment duration $\sim 20-30$ minutes). These data include 3 human and 4 rat ventricles, for which both pressure and volume were recorded at each isoproterenol dose (Fig. 3b). Intraventricular pressure and volume were measured simultaneously using a Millar MPVS-Ultra single segment foundation system for rats with SPR-869NR rat pressure-volume (PV) catheters (ADInstruments, Colorado Springs, CO). Catheter calibration and PV experiments were performed in freshly prepared Tyrode's solution. First, tissue-engineered ventricles were transferred to a $3.5 \mathrm{~cm}$ petri dish mounted on a temperature-controlled stage (Warner Instruments, Hamden CT) to maintain $35{ }^{\circ} \mathrm{C}$ working temperature. Ventricles were sutured to silicone tubing through which catheters were inserted (Supplementary Fig. 4A). Data 
acquired at a sampling rate of 2,000 samples per second using manufacturer-supplied acquisition systems (Millar MPVS ultra, ADInstruments LabChart) and was exported for post-processing with custom Matlab scripts (MathWorks, Natick MA). Catheter calibration. Catheters were equilibrated at $35^{\circ} \mathrm{C}$ prior to use and calibrated for pressure using a manometer (Meriam M2; Meriam Process Technologies, Cleveland, $\mathrm{OH}$ ) and volume using manufacturer-supplied cuvettes (Millar P/N 910-1048). We observed a non-linear conductance/volume catheter response (Supplementary Fig. 4B.i), as expected for these systems ${ }^{82}$. We performed separate scaffold-specific calibrations, where cell-free scaffolds were inflated and deflated by manual application of pressure through a solution-filled syringe connected to the ventricle through a junction port. Here, we aimed to validate our use of thin PCL-gelatin scaffold walls in place of polycarbonate cuvettes or murine myocardium for which the system and catheters were designed and optimized ${ }^{24,82-86}$. Volume estimated by calculating the slope of measured conductance versus cuvette volume (Supplementary Fig. 4B.i) or by scaffold filling (Supplementary Fig. 4B.ii) were in good agreement within a relevant working volume range of $\sim 300-600 \mu \mathrm{L}$. We then used a precision pressure-driven microfluidic pump (Elveflow OB1, Elvesys, Paris France) to generate small-amplitude pressure variations applied to a model ventricle scaffold through the extra-ventricular (assist) channel of our custom-made bioreactor (Supplementary Figures S5). The bioreactor is described in more detail in Supplementary Figures S8 and S9. By generating successively smaller peak-to-peak pressure sinusoids, we verified that pressure and volume variations generated by our tissue engineered model ventricles were above the noise floor (Supplementary Figs. S5-S7). In some cases, pressure or volume signal noise and drift were reduced by post-processing using Matlab's smooth and polyfit functions (Supplementary Fig. 4C). Pressure and volume signals were normalized by polynomial background subtraction to reduce unwanted signal drift from large data sets, clarifying frequency-domain analysis of ventricle beat rates over these time periods (Supplementary Fig. 5). $\beta$-adrenergic response of tissue-engineered ventricles. The contractile response of tissue-engineered ventricles to increasing concentrations of the $\beta$-adrenergic agonist isoproterenol was measured as follows. A $100 \mathrm{mM}$ stock solution of isoproterenol (SigmaAldrich) containing $110 \mu \mathrm{M}$ ascorbic acid (Sigma-Aldrich) and $45 \mu \mathrm{M}$ EDTA (SigmaAldrich) was prepared in Tyrode's solution and stored at $-20^{\circ} \mathrm{C}$. Working concentrations were prepared fresh for each experiment by serial 10-fold dilution and kept on ice and protected from light during experiments. The engineered tissues were exposed to concentrations of isoproterenol ranging from $1 \mathrm{e}^{-10} \mathrm{M}$ to $1 \mathrm{e}^{-4} \mathrm{M}$ by cumulative addition of $1.0 \log$ doses every 2 minutes. Pressure and volume recordings collected from the last 30 seconds of each measurement interval were converted to the frequency domain by Fast Fourier Transform (FFT) using Matlab and beat rates were estimated using Matlab's findpeaks function applied to pressure or volume FFT data (Supplementary Fig. 5).

\section{Heart Bioreactor (HBR) Design and Fabrication}

HBR designs were created using SolidWorks (DS SolidWorks Corporation, Waltham MA). HBR components were fabricated by rapid manufacturing providers (Proto labs, Maple Plain, MN) and by the staff of the John A. Paulson School of Engineering and Applied Sciences (SEAS) Scientific Instrument Shop at Harvard University. The HBR body consisted of three separated polycarbonate pieces that were assembled using stainless steel 
screws and silicone gaskets (Fig. 4A, Supplementary Figs. 6,7). To increase optical transparency of HBR polycarbonate components, we performed post-fabrication vapor-

polishing in our laboratory by methylene chloride vapor exposure in a chemical fume hood.

\section{Echocardiography}

We acquired echocardiographic images and videos of ventricle contraction using a Vevo 2100 system (VisualSonics, Toronto, Canada) at the Boston Children's Hospital Small Animal Imaging Laboratory (BCH-SAIL). We used a $21 \mathrm{MHz}$ probe $(\sim 60 \mu \mathrm{m}$ resolution, $\sim 1.4 \mathrm{~cm}$ imaging depth) coupled to the HBR window through an index-matching gel.

\section{Statistical analysis}

For analysis of tissue OOP and beat rate response to isoproterenol, one-way ANOVA between the compositional groups were conducted using SigmaPlot (v13.0, Systat Software Inc.). For pairwise comparison, the Tukey test was applied. For all statistical analyses, pvalues less than 0.05 were considered statistically significant.

\section{Code availability}

The custom MATLAB-based optical mapping analysis package is available at https:// code.google.com/archive/p/rhythmanalysis-software. Custom Matlab scripts used for data smoothing, Fourier transformation and plotting are available upon request.

\section{Data availability}

All data generated and analysed during this study are included in the paper and its Supplementary Information.

\section{Supplementary Material}

Refer to Web version on PubMed Central for supplementary material.

\section{Acknowledgements}

This work was sponsored by the John A. Paulson School of Engineering and Applied Sciences at Harvard University, the Wyss Institute for Biologically Inspired Engineering at Harvard University, Harvard Materials Research Science and Engineering Center grant DMR-1420570, Defense Threat Reduction Agency (DTRA) subcontract \#312659 from Los Alamos National Laboratory under a prime DTRA contract no. DEAC52-06NA25396, and the National Center for Advancing Translational Sciences of the National Institutes of Health under Award Numbers UH3TR000522 and 1-UG3-HL-141798-01. The content is solely the responsibility of the authors and does not necessarily represent the official views of the National Institutes of Health. This work was supported in part by the U. S. Army Research Laboratory and the U. S. Army Research Office under Contract No. W911NF-12-2-0036. The views and conclusions contained in this document are those of the authors and should not be interpreted as representing the official policies, either expressed or implied, of the Army Research Office, Army Research Laboratory, or the U.S. Government. The U.S. Government is authorized to reproduce and distribute reprints for Government purposes notwithstanding any copyright notation hereon. This work was performed in part at the Center for Nanoscale Systems (CNS), a member of the National Nanotechnology Coordinated Infrastructure Network (NNCI), which is supported by the National Science Foundation under NSF award no. 1541959. CNS is part of Harvard University. We thank Michael McKenna and the staff at Harvard University's John A. Paulson School of Engineering and Applied Sciences Scientific Instrument Shop for manufacturing heart bioreactor and nanofiber production system components. We thank Matthew Griswold and Alexander Cho for technical assistance, Jacques Guyette and Harald Ott for providing decellularized human left ventricle myocardial tissue samples, Erin Snay for assistance with echocardiographic imaging at the Boston Children's Hospital Small Animal Imaging Laboratory, and Michael Rosnach for assistance with photography and illustrations. We thank Prof. Andre Kleber for his expertise and insightful discussions. 


\section{References}

1. Benam KH et al. Engineered in vitro disease models. Annu Rev Pathol 10, 195-262, doi:10.1146/ annurev-pathol-012414-040418 (2015). [PubMed: 25621660]

2. Tzatzalos E, Abilez OJ, Shukla P \& Wu JC Engineered heart tissues and induced pluripotent stem cells: Macro- and microstructures for disease modeling, drug screening, and translational studies. Adv Drug Deliv Rev 96, 234-244, doi:10.1016/j.addr.2015.09.010 (2016). [PubMed: 26428619]

3. Pacher P, Nagayama T, Mukhopadhyay P, Batkai S \& Kass DA Measurement of cardiac function using pressure-volume conductance catheter technique in mice and rats. Nat Protoc 3, 1422-1434, doi:10.1038/nprot.2008.138 (2008). [PubMed: 18772869]

4. Ram R, Mickelsen DM, Theodoropoulos C \& Blaxall BC New approaches in small animal echocardiography: imaging the sounds of silence. American journal of physiology. Heart and circulatory physiology 301, H1765-1780, doi:10.1152/ajpheart.00559.2011 (2011). [PubMed: 21873501]

5. Bakermans AJ et al. Small animal cardiovascular MR imaging and spectroscopy. Progress in nuclear magnetic resonance spectroscopy 88-89, 1-47, doi:10.1016/j.pnmrs.2015.03.001 (2015). [PubMed: 26282195]

6. Chandrasekera PC \& Pippin JJ The human subject: an integrative animal model for 21(st) century heart failure research. Am J Transl Res 7, 1636-1647 (2015). [PubMed: 26550463]

7. Gloschat CR et al. Arrhythmogenic and metabolic remodelling of failing human heart. J Physiol 594, 3963-3980, doi:10.1113/JP271992 (2016). [PubMed: 27019074]

8. Karakikes I, Ameen M, Termglinchan V \& Wu JC Human induced pluripotent stem cell-derived cardiomyocytes: Insights into molecular, cellular, and functional phenotypes. Circ Res 117, 80-88, doi:10.1161/Circresaha.117.305365 (2015). [PubMed: 26089365]

9. Feric NT \& Radisic M Maturing human pluripotent stem cell-derived cardiomyocytes in human engineered cardiac tissues. Adv Drug Deliv Rev 96, 110-134, doi:10.1016/j.addr.2015.04.019 (2016). [PubMed: 25956564]

10. Eder A, Vollert I, Hansen A \& Eschenhagen T Human engineered heart tissue as a model system for drug testing. Adv Drug Deliver Rev 96, 214-224, doi:10.1016/j.addr.2015.05.010 (2016).

11. Wang $\mathrm{G}$ et al. Modeling the mitochondrial cardiomyopathy of Barth syndrome with induced pluripotent stem cell and heart-on-chip technologies. Nat Med 20, 616-623, doi:10.1038/nm.3545 (2014). [PubMed: 24813252]

12. Lind JU et al. Instrumented cardiac microphysiological devices via multimaterial threedimensional printing. Nat Mater, doi:10.1038/nmat4782 (2016).

13. Boudou $\mathrm{T}$ et al. A microfabricated platform to measure and manipulate the mechanics of engineered cardiac microtissues. Tissue engineering. Part A 18, 910-919, doi:10.1089/ten.TEA. 2011.0341 (2012). [PubMed: 22092279]

14. Nunes SS et al. Biowire: a platform for maturation of human pluripotent stem cell-derived cardiomyocytes. Nat Methods 10, 781-+, doi:10.1038/Nmeth.2524 (2013). [PubMed: 23793239]

15. Thavandiran $\mathrm{N}$ et al. Design and formulation of functional pluripotent stem cell-derived cardiac microtissues. Proceedings of the National Academy of Sciences of the United States of America 110, E4698-4707, doi:10.1073/pnas.1311120110 (2013). [PubMed: 24255110]

16. Mannhardt I et al. Human engineered heart tissue: Analysis of contractile force. Stem Cell Reports 7, 29-42, doi:10.1016/j.stemcr.2016.04.011 (2016). [PubMed: 27211213]

17. Huebsch N et al. Miniaturized iPS-cell-derived cardiac muscles for physiologically relevant drug response analyses. Sci Rep 6, 24726, doi:10.1038/srep24726 (2016). [PubMed: 27095412]

18. Mathur A et al. Human iPSC-based cardiac microphysiological system for drug screening applications. Sci Rep 5, 8883, doi:10.1038/srep08883 (2015). [PubMed: 25748532]

19. Turnbull IC et al. Advancing functional engineered cardiac tissues toward a preclinical model of human myocardium. FASEB J 28, 644-654, doi:10.1096/fj.13-228007 (2014). [PubMed: 24174427]

20. Sidorov VY et al. I-Wire Heart-on-a-Chip I: Three-dimensional cardiac tissue constructs for physiology and pharmacology. Acta Biomater 48, 68-78, doi:10.1016/j.actbio.2016.11.009 (2017). [PubMed: 27818308] 
21. Godier-Furnemont AFG et al. Physiologic force-frequency response in engineered heart muscle by electromechanical stimulation. Biomaterials 60, 82-91, doi:10.1016/j.biomaterials.2015.03.055 (2015). [PubMed: 25985155]

22. Tiburcy $\mathrm{M}$ et al. Defined engineered human myocardium with advanced maturation for applications in heart failure modelling and repair. Circulation, doi:10.1161/CIRCULATIONAHA. 116.024145 (2017).

23. Mathur A, Ma Z, Loskill P, Jeeawoody S \& Healy KE In vitro cardiac tissue models: Current status and future prospects. Adv Drug Deliv Rev 96, 203-213, doi:10.1016/j.addr.2015.09.011 (2016). [PubMed: 26428618]

24. Pacher P, Nagayama T, Mukhopadhyay P, Batkai S \& Kass DA Measurement of cardiac function using pressure-volume conductance catheter technique in mice and rats. Nat Protoc 3, 1422-1434 (2008). [PubMed: 18772869]

25. Burkhoff D, Mirsky I \& Suga H Assessment of systolic and diastolic ventricular properties via pressure-volume analysis: a guide for clinical, translational, and basic researchers. American journal of physiology. Heart and circulatory physiology 289, H501-512, doi:10.1152/ajpheart. 00138.2005 (2005). [PubMed: 16014610]

26. Lee EJ, Kim do E, Azeloglu EU \& Costa KD Engineered cardiac organoid chambers: toward a functional biological model ventricle. Tissue engineering. Part A 14, 215-225, doi:10.1089/tea. 2007.0351 (2008). [PubMed: 18333774]

27. Gonen-Wadmany M, Gepstein L \& Seliktar D Controlling the cellular organization of tissueengineered cardiac constructs. Ann N Y Acad Sci 1015, 299-311, doi:10.1196/annals.1302.025 (2004). [PubMed: 15201169]

28. Yildirim Y et al. Development of a biological ventricular assist device: preliminary data from a small animal model. Circulation 116, I16-23, doi:10.1161/CIRCULATIONAHA.106.679688 (2007). [PubMed: 17846298]

29. Li RA et al. Bioengineering an electro-mechanically functional miniature ventricular heart chamber from human pluripotent stem cells. Biomaterials, doi:10.1016/j.biomaterials.2018.02.024 (2018).

30. Costa KD, Takayama Y, McCulloch AD \& Covell JW Laminar fiber architecture and threedimensional systolic mechanics in canine ventricular myocardium. The American journal of physiology 276, H595-607 (1999). [PubMed: 9950861]

31. Arts T, Costa KD, Covell JW \& McCulloch AD Relating myocardial laminar architecture to shear strain and muscle fiber orientation. American journal of physiology. Heart and circulatory physiology 280, H2222-2229 (2001). [PubMed: 11299225]

32. Rohr S, Scholly DM \& Kleber AG Patterned growth of neonatal rat heart cells in culture. Morphological and electrophysiological characterization. Circ Res 68, 114-130 (1991). [PubMed: 1984856]

33. Kleber AG \& Rudy Y Basic mechanisms of cardiac impulse propagation and associated arrhythmias. Physiol Rev 84, 431-488, doi:10.1152/physrev.00025.2003 (2004). [PubMed: 15044680]

34. Bursac N, Parker KK, Iravanian S \& Tung L Cardiomyocyte cultures with controlled macroscopic anisotropy: a model for functional electrophysiological studies of cardiac muscle. Circ Res 91, e45-54 (2002). [PubMed: 12480825]

35. Feinberg AW et al. Controlling the contractile strength of engineered cardiac muscle by hierarchal tissue architecture. Biomaterials 33, 5732-5741, doi:10.1016/j.biomaterials.2012.04.043 (2012). [PubMed: 22594976]

36. Zong X et al. Electrospun fine-textured scaffolds for heart tissue constructs. Biomaterials 26, 5330-5338, doi:10.1016/j.biomaterials.2005.01.052 (2005). [PubMed: 15814131]

37. Kai D, Prabhakaran MP, Jin G \& Ramakrishna S Guided orientation of cardiomyocytes on electrospun aligned nanofibers for cardiac tissue engineering. J Biomed Mater Res B Appl Biomater 98, 379-386, doi:10.1002/jbm.b.31862 (2011). [PubMed: 21681953]

38. Kenar H, Kose GT, Toner M, Kaplan DL \& Hasirci VA 3D aligned microfibrous myocardial tissue construct cultured under transient perfusion. Biomaterials 32, 5320-5329, doi:10.1016/ j.biomaterials.2011.04.025 (2011). [PubMed: 21570112] 
39. Orlova Y, Magome N, Liu L, Chen Y \& Agladze K Electrospun nanofibers as a tool for architecture control in engineered cardiac tissue. Biomaterials 32, 5615-5624, doi:10.1016/ j.biomaterials.2011.04.042 (2011). [PubMed: 21600646]

40. Capulli AK, MacQueen LA, Sheehy SP \& Parker KK Fibrous scaffolds for building hearts and heart parts. Adv Drug Deliv Rev 96, 83-102, doi:10.1016/j.addr.2015.11.020 (2016). [PubMed: 26656602]

41. Mauck RL et al. Engineering on the straight and narrow: the mechanics of nanofibrous assemblies for fiber-reinforced tissue regeneration. Tissue Eng Part B Rev 15, 171-193, doi:10.1089/ten.TEB. 2008.0652 (2009). [PubMed: 19207040]

42. Pope AJ, Sands GB, Smaill BH \& LeGrice IJ Three-dimensional transmural organization of perimysial collagen in the heart. Am J Physiol-Heart C 295, H1243-H1252, doi:10.1152/ajpheart. 00484.2008 (2008).

43. Sheehy SP, Grosberg A \& Parker KK The contribution of cellular mechanotransduction to cardiomyocyte form and function. Biomechanics and modeling in mechanobiology 11, 12271239, doi:10.1007/s10237-012-0419-2 (2012). [PubMed: 22772714]

44. Kim DH et al. Nanoscale cues regulate the structure and function of macroscopic cardiac tissue constructs. Proceedings of the National Academy of Sciences of the United States of America 107, 565-570, doi:10.1073/pnas.0906504107 (2010). [PubMed: 20018748]

45. Savadjiev $P$ et al. Heart wall myofibers are arranged in minimal surfaces to optimize organ function. Proceedings of the National Academy of Sciences of the United States of America 109, 9248-9253, doi:10.1073/pnas.1120785109 (2012). [PubMed: 22645368]

46. Deravi LF et al. Design and fabrication of fibrous nanomaterials using pull spinning. Macromolecular Materials and Engineering 302, 1600404, doi:10.1002/mame.201600404 (2017).

47. Ruoslahti E RGD and other recognition sequences for integrins. Annu Rev Cell Dev Biol 12, $697-$ 715, doi:10.1146/annurev.cellbio.12.1.697 (1996). [PubMed: 8970741]

48. Katagiri Y, Brew SA \& Ingham KC All six modules of the gelatin-binding domain of fibronectin are required for full affinity. J Biol Chem 278, 11897-11902, doi:10.1074/jbc.M212512200 (2003). [PubMed: 12538576]

49. Meiry $\mathrm{G}$ et al. Evolution of action potential propagation and repolarization in cultured neonatal rat ventricular myocytes. J Cardiovasc Electrophysiol 12, 1269-1277 (2001). [PubMed: 11761415]

50. Morse PM \& Feshbach H Methods of theoretical physics. (McGraw-Hill, 1953).

51. Mandegar MA et al. CRISPR interference efficiently induces specific and reversible gene silencing in human iPSCs. Cell stem cell, doi:10.1016/j.stem.2016.01.022 (2016).

52. Rohr S, Kucera JP \& Kleber AG Slow conduction in cardiac tissue, I: effects of a reduction of excitability versus a reduction of electrical coupling on microconduction. Circ Res 83, 781-794 (1998). [PubMed: 9776725]

53. Yang XL, Pabon L \& Murry CE Engineering Adolescence Maturation of Human Pluripotent Stem Cell-Derived Cardiomyocytes. Circulation research 114, 511-523, doi:10.1161/Circresaha. 114.300558 (2014). [PubMed: 24481842]

54. Pasqualini FS, Sheehy SP, Agarwal A, Aratyn-Schaus Y \& Parker KK Structural phenotyping of stem cell-derived cardiomyocytes. Stem Cell Reports 4, 340-347, doi:10.1016/j.stemcr. 2015.01.020 (2015). [PubMed: 25733020]

55. Akselrod S et al. Power spectrum analysis of heart rate fluctuation: a quantitative probe of beat-tobeat cardiovascular control. Science 213, 220-222 (1981). [PubMed: 6166045]

56. Fenske $S$ et al. Comprehensive multilevel in vivo and in vitro analysis of heart rate fluctuations in mice by ECG telemetry and electrophysiology. Nat Protoc 11, 61-86, doi:10.1038/nprot.2015.139 (2016). [PubMed: 26658468]

57. Barrett AM \& Carter J Comparative chronotropic activity of beta-adrenoceptive antagonists. Br J Pharmacol 40, 373-381 (1970). [PubMed: 4395742]

58. Brito-Martins M, Harding SE \& Ali NN beta(1)- and beta(2)-adrenoceptor responses in cardiomyocytes derived from human embryonic stem cells: comparison with failing and nonfailing adult human heart. Br J Pharmacol 153, 751-759, doi:10.1038/sj.bjp.0707619 (2008). [PubMed: 18193079] 
59. Simpson P \& Savion S Differentiation of rat myocytes in single cell cultures with and without proliferating nonmyocardial cells. Cross-striations, ultrastructure, and chronotropic response to isoproterenol. Circ Res 50, 101-116 (1982). [PubMed: 7053872]

60. Moretti A et al. Patient-specific induced pluripotent stem-cell models for long-QT syndrome. N Engl J Med 363, 1397-1409, doi:10.1056/NEJMoa0908679 (2010). [PubMed: 20660394]

61. Koglin J, Bohm M, Vonscheidt W, Stablein A \& Erdmann E Antiadrenergic effect of carbachol but not of adenosine on contractility in the intact human ventricle in-vivo. J Am Coll Cardiol 23, 678683 (1994). [PubMed: 8113552]

62. Lim ZY, Maskara B, Aguel F, Emokpae R Jr. \& Tung L Spiral wave attachment to millimeter-sized obstacles. Circulation 114, 2113-2121, doi:10.1161/CIRCULATIONAHA.105.598631 (2006). [PubMed: 17088465]

63. Ogle BM et al. Distilling complexity to advance cardiac tissue engineering. Sci Transl Med 8, 342ps313, doi:10.1126/scitranslmed.aad2304 (2016).

64. Feinberg AW et al. Muscular thin films for building actuators and powering devices. Science 317 , 1366-1370, doi:10.1126/science.1146885 (2007). [PubMed: 17823347]

65. Novosel EC, Kleinhans C \& Kluger PJ Vascularization is the key challenge in tissue engineering. Adv Drug Deliver Rev 63, 300-311, doi:10.1016/j.addr.2011.03.004 (2011).

66. Lundberg MS, Baldwin JT \& Buxton DB Building a bioartificial heart: Obstacles and opportunities. J Thorac Cardiovasc Surg 153, 748-750, doi:10.1016/j.jtcvs.2016.10.103 (2017). [PubMed: 28139212]

67. Chaturvedi RR et al. Passive stiffness of myocardium from congenital heart disease and implications for diastole. Circulation 121, 979-988, doi:10.1161/CIRCULATIONAHA. 109.850677 (2010). [PubMed: 20159832]

68. Quinn KP et al. Optical metrics of the extracellular matrix predict compositional and mechanical changes after myocardial infarction. Sci Rep 6, 35823, doi:10.1038/srep35823 (2016). [PubMed: 27819334]

69. Gonzalez GM et al. Production of Synthetic, Para-Aramid and Biopolymer Nanofibers by Immersion Rotary Jet-Spinning. Macromolecular Materials and Engineering 302, doi:ARTN 1600365 10.1002/mame.201600365 (2017).

70. Gladman AS, Matsumoto EA, Nuzzo RG, Mahadevan L \& Lewis JA Biomimetic 4D printing. Nat Mater 15, 413-418, doi:10.1038/nmat4544 (2016). [PubMed: 26808461]

71. Zimmermann WH et al. Three-dimensional engineered heart tissue from neonatal rat cardiac myocytes. Biotechnol Bioeng 68, 106-114 (2000). [PubMed: 10699878]

72. Germanguz I et al. Molecular characterization and functional properties of cardiomyocytes derived from human inducible pluripotent stem cells. J Cell Mol Med 15, 38-51, doi:10.1111/j. 1582-4934.2009.00996.x (2011). [PubMed: 20041972]

73. Ronaldson-Bouchard $\mathrm{K}$ et al. Advanced maturation of human cardiac tissue grown from pluripotent stem cells. Nature 556, 239-243, doi:10.1038/s41586-018-0016-3 (2018). [PubMed: 29618819]

74. Endoh M Force-frequency relationship in intact mammalian ventricular myocardium: physiological and pathophysiological relevance. Eur J Pharmacol 500, 73-86, doi:10.1016/j.ejphar.2004.07.013 (2004). [PubMed: 15464022]

75. Bai SL, Campbell SE, Moore JA, Morales MC \& Gerdes AM Influence of age, growth, and sex on cardiac myocyte size and number in rats. Anat Rec 226, 207-212, doi:10.1002/ar.1092260210 (1990). [PubMed: 2301738]

76. Feric NT \& Radisic M Strategies and challenges to myocardial replacement therapy. Stem Cells Transl Med 5, 410-416, doi:10.5966/sctm.2015-0288 (2016). [PubMed: 26933042]

77. Pecha S, Eschenhagen $\mathrm{T} \&$ Reichenspurner H Myocardial tissue engineering for cardiac repair. J Heart Lung Transplant 35, 294-298, doi:10.1016/j.healun.2015.12.007 (2016). [PubMed: 26856673]

78. Klabunde RE Cardiovascular physiology concepts. 2nd edn, (Lippincott Williams \& Wilkins/ Wolters Kluwer, 2012).

79. Park SJ et al. Phototactic guidance of a tissue-engineered soft-robotic ray. Science 353, 158-162, doi:10.1126/science.aaf4292 (2016). [PubMed: 27387948] 
80. Guyette JP et al. Bioengineering human myocardium on native extracellular matrix. Circ Res 118, 56-72, doi:10.1161/CIRCRESAHA.115.306874 (2016). [PubMed: 26503464]

81. Laughner JI, Ng FS, Sulkin MS, Arthur RM \& Efimov IR Processing and analysis of cardiac optical mapping data obtained with potentiometric dyes. Am J Physiol-Heart C 303, H753-H765, doi:10.1152/ajpheart.00404.2012 (2012).

82. Pearce JA, Porterfield JE, Larson ER, Valvano JW \& Feldman MD Accuracy considerations in catheter based estimation of left ventricular volume. Conf Proc IEEE Eng Med Biol Soc 2010, 3556-3558, doi:10.1109/IEMBS.2010.5627712 (2010). [PubMed: 21097041]

83. Baan $\mathrm{J}$ et al. Continuous stroke volume and cardiac output from intra-ventricular dimensions obtained with impedance catheter. Cardiovasc Res 15, 328-334 (1981). [PubMed: 7296590]

84. Baan J et al. Continuous measurement of left ventricular volume in animals and humans by conductance catheter. Circulation 70, 812-823 (1984). [PubMed: 6386218]

85. Raghavan $\mathrm{K}$ et al. Electrical conductivity and permittivity of murine myocardium. IEEE Trans Biomed Eng 56, 2044-2053, doi:10.1109/TBME.2009.2012401 (2009). [PubMed: 19605306]

86. Clark JE \& Marber MS Advancements in pressure-volume catheter technology - stress remodelling after infarction. Exp Physiol 98, 614-621, doi:10.1113/expphysiol.2012.064733 (2013). [PubMed: 23064506] 
a

$\begin{array}{ll}\text { Heart Ventricle ECM-inspired Scaffold } & \text { Measurement } \\ \text { Heart Catheterization }\end{array}$

b

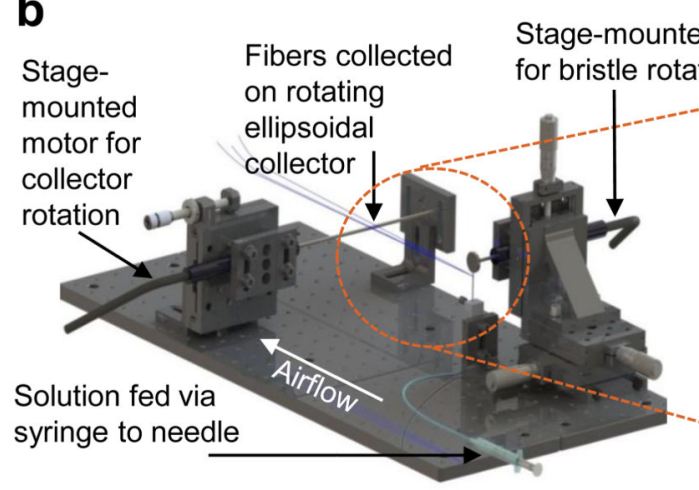

C

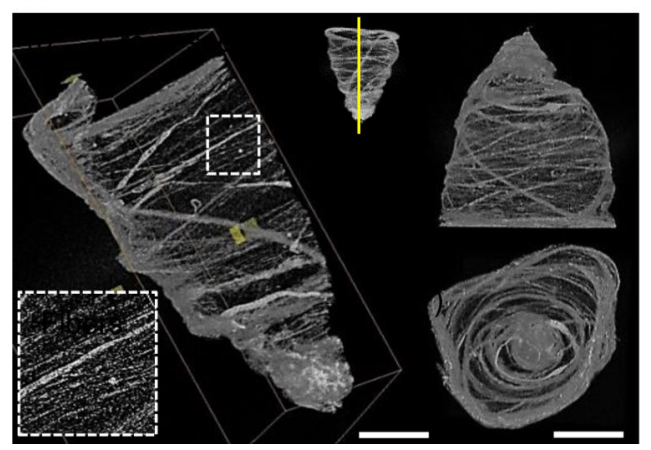

d

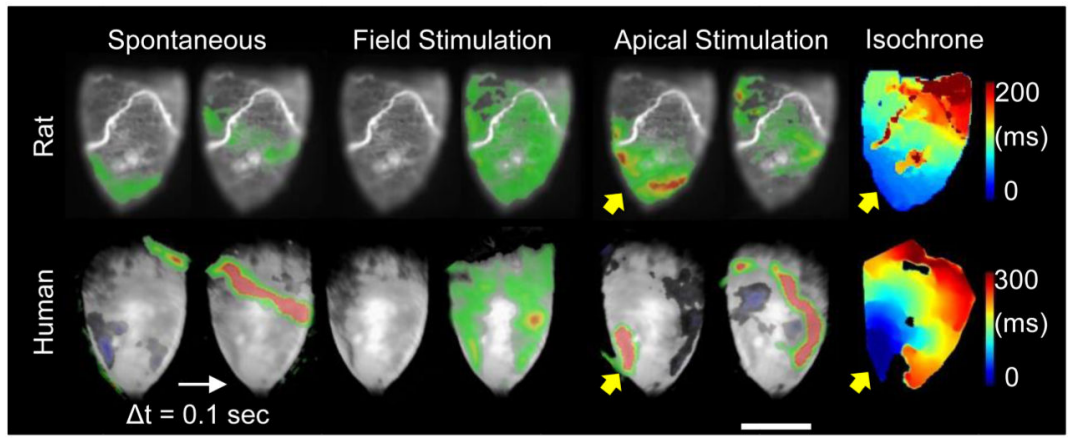

Figure 1|. Tissue-engineered model ventricles recapitulate key structural and functional aspects of natural ventricular myocardium.

a, A schematic overview of the ventricle design-build-test project phases, from left to right: Human left ventricle ellipsoidal shape and fibrous extra-cellular matrix (ECM) inspired our use of circumferentially oriented nanofibers in scale-model ellipsoidal ventricle scaffolds. We seeded these scaffolds with cardiomyocytes to produce tissue-engineered ventricles and evaluated their contractile strength by pressure-volume catheterization. b, Pull spinning a nanofibrous ellipsoidal model ventricle scaffold: A high-speed rotating bristle dips into a fixed, continuous polymer source that is fed via a syringe pump through a small orifice (in this case a needle). The bristle pulls the polymer column into a polymer jet that is ejected towards a rotating collector. Solvent evaporation occurs as the polymer jet travels towards the collection mandrel, where the resulting nanofibers are collected. The resulting ventricle scaffolds are removed from the collector by tweezers in a hydration bath. c, Micro-computed tomography $(\mu \mathrm{CT})$ images of a ventricle scaffold. $\mathbf{d}$, Calcium propagation imaging performed on day 14. Spontaneous activity produced propagating waves but they did not always originate at the apex (left). Field stimulation, resulting from electrical pacing where 
electrodes were placed far from the ventricle surface, produced uniform calcium activation (middle). Apical point stimulation (indicated by yellow arrows) produced calcium waves that propagated from apex to base at a rate of $9.33 \mathrm{~cm} / \mathrm{sec}$ for neonatal rat ventricular myocyte (NRVM) ventricles and $5.2 \mathrm{~cm} / \mathrm{sec}$ for human induced pluripotent stem cell-derived cardiomyocyte (hiPSC-CM) ventricles (right). Calcium fluorescence intensity is displayed as a heat map ranging from blue (min) to red (max), overlaid on a greyscale image of the ventricle surface 
a Tissue-engineered model ventricle immunostaining

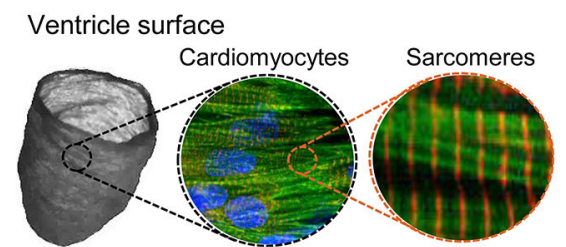

Ventricle cross-section

b
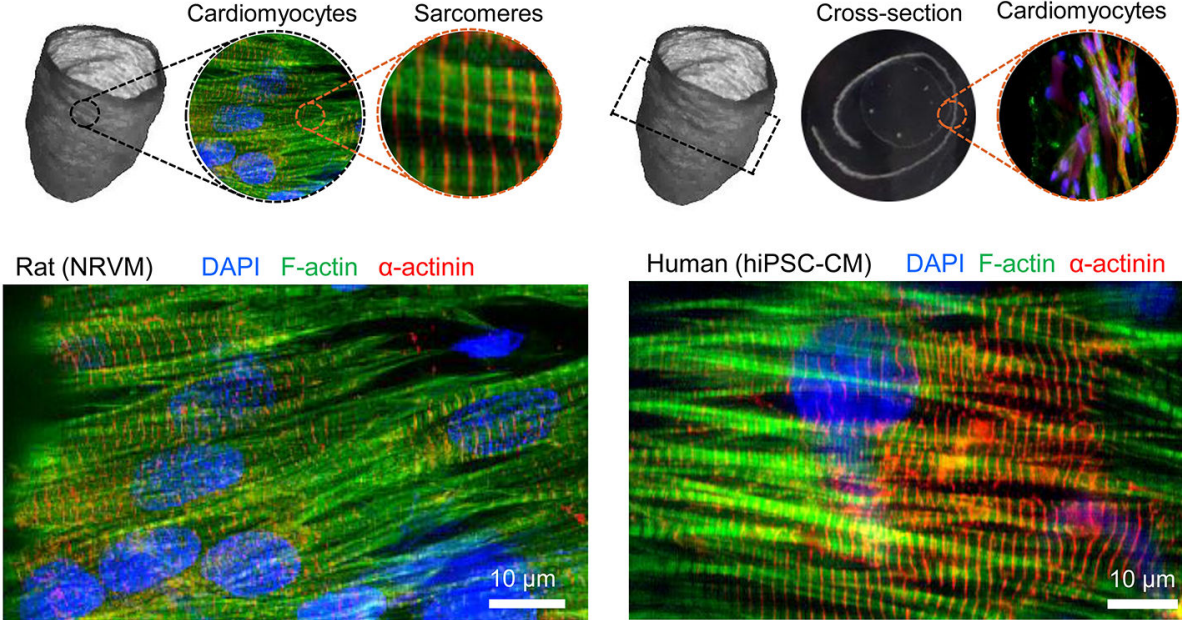

造
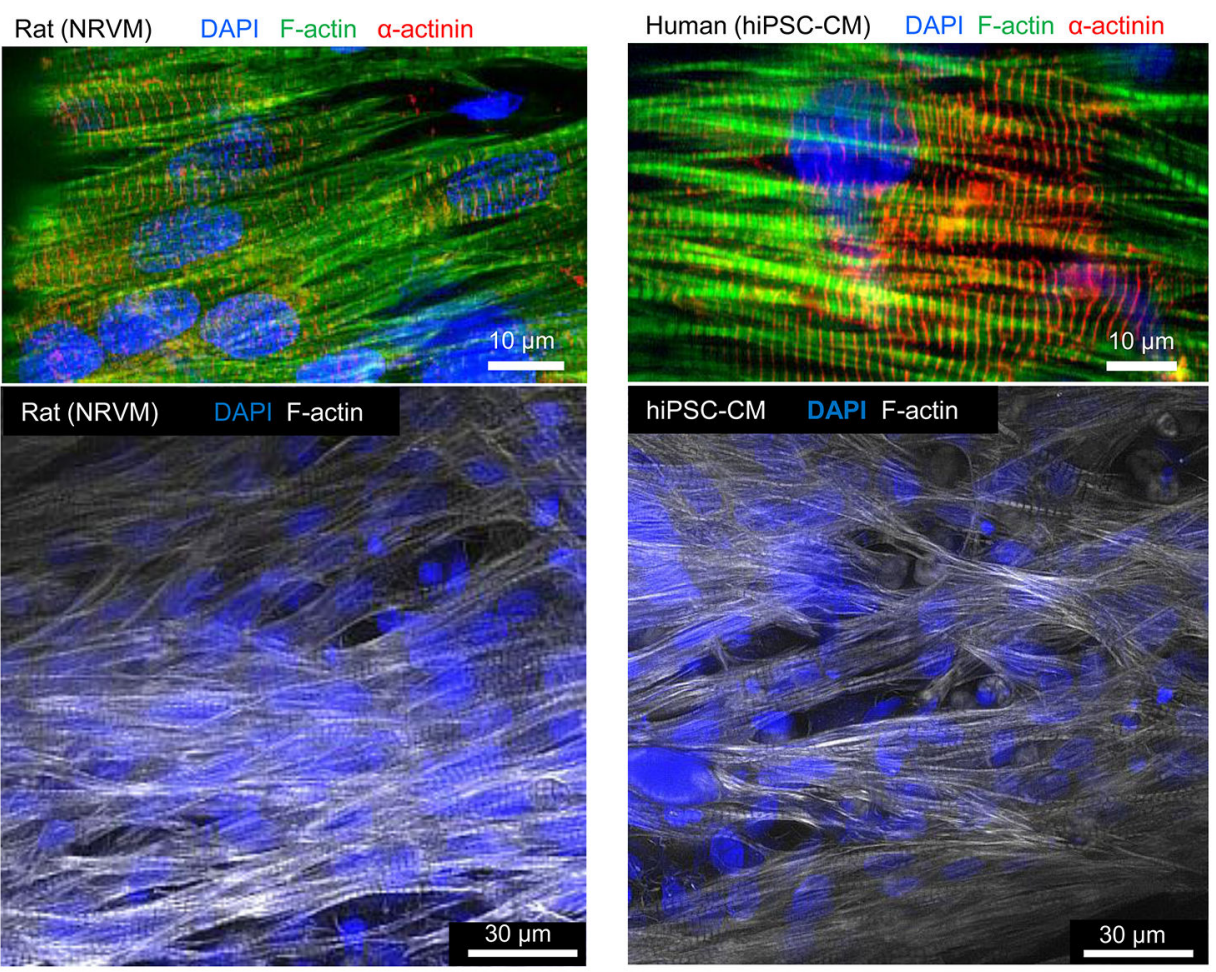

C
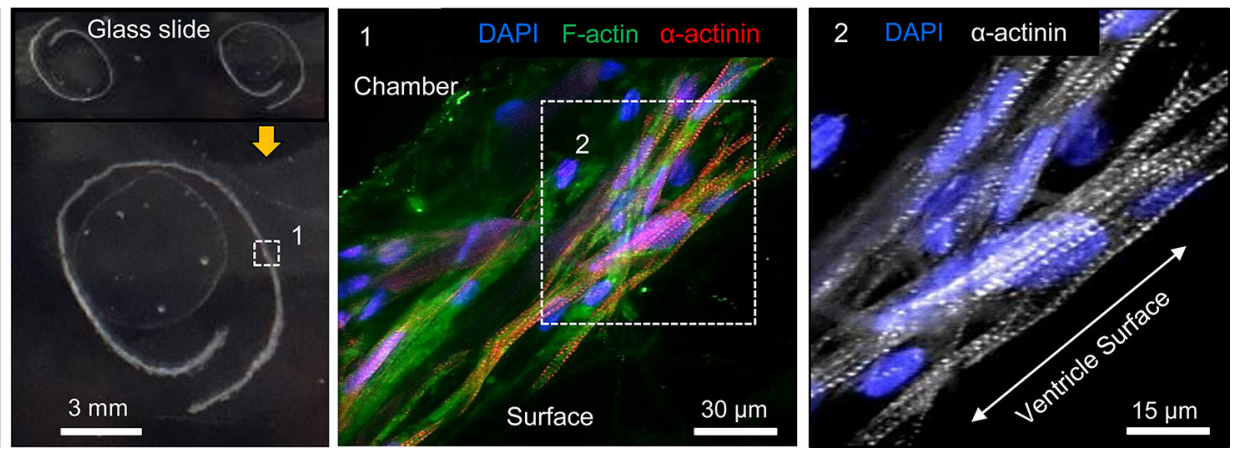

Figure 2|. Tissue-engineered ventricle immunostaining.

a, A schematic of ventricle surface or cross-section immunostains shown in $\mathbf{b}$ (surface) and $\mathbf{c}$ (cross-section). In all cases, cells were introduced via the exterior surface and imaging was performed following 14 days in culture. Immunostaining confirmed that both neonatal rat ventricular myocytes (NRVM) and human induced pluripotent stem cell-derived cardiomyocytes (Cor.4U hiPSC-CM) infiltrated the scaffolds and were aligned roughly circumferentially, coincident with the scaffold's nanofiber ultrastructure. 
a Tissue engineered model ventricle catheterization
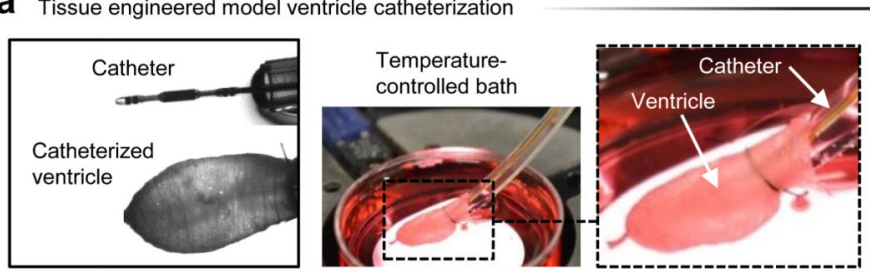

Intra-ventricular pressure and volume measurements

b Intra-ventricular pressure, $\mathrm{P}$, and volume, $\mathrm{V}$
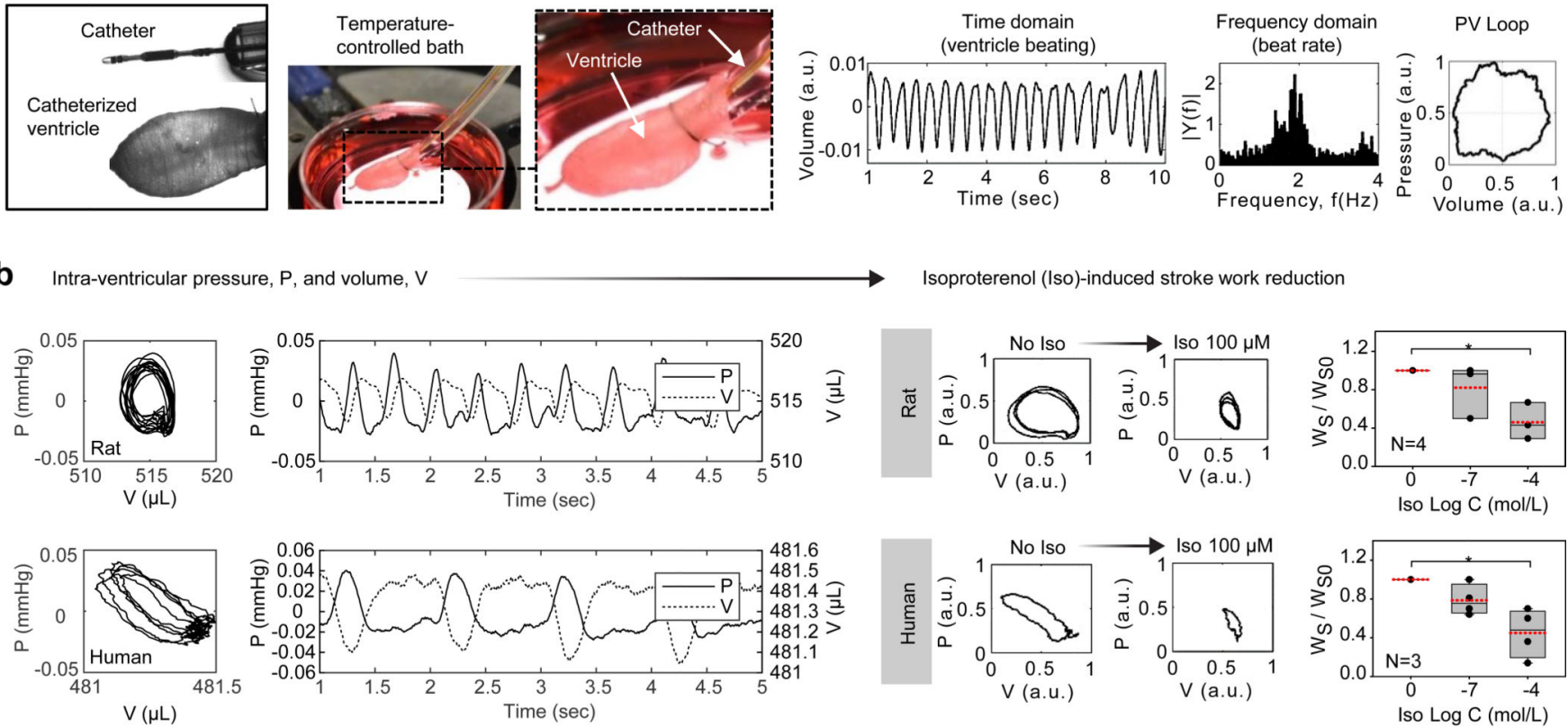

C

Isoproterenol (Iso)-dependent beat rates for rat $(\mathrm{N}=8)$ and human hiPSC-CM $(\mathrm{N}=4)$ engineered model ventricle chambers
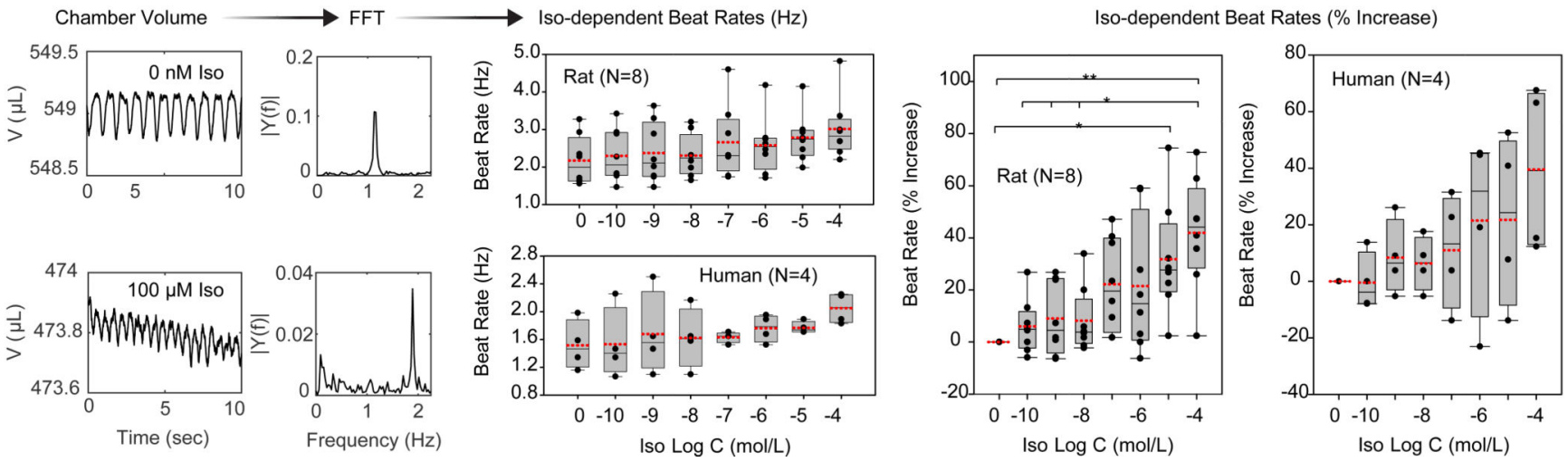

Figure 3|. Intra-ventricular pressure-volume (PV) data obtained by tissue-engineered ventricle catheterization.

a, An overview of the ventricle catheterization procedure. Catheters were fed through tubing on which the ventricle base was sutured. Ventricles were submerged within a $3.5 \mathrm{~cm}$ petri dish bath, which was mounted on a temperature-controlled heating stage. Catheter readouts fed to signal amplification instruments provided real-time measurements of intra-ventricular pressure and volume. $\mathbf{b}$, Intra-ventricular pressure, $\mathrm{P}$, and volume, $\mathrm{V}$, measured by catheterization of neonatal rat ventricular myocyte (NRVM)- or human induced pluripotent stem cell-derived cardiomyocyte (Cor.4U hiPSC-CM)-based ventricles. Exposure to isoproterenol (Iso) reduced stroke work of both rat and human ventricles. Here, P and V were normalized by polynomial fit to remove measurement drift occurring over the course of multiple Iso doses. $\mathrm{P}$-values were $\mathrm{P}=0.04$ (NRVM, $\mathrm{N}=4$ ventricles) and $\mathrm{P}=0.038$ (Cor.4U, $\mathrm{N}=3$ ventricles). c, Iso-dependent beat rates for rat $(\mathrm{N}=8$ ventricles) and human $(\mathrm{N}=4$ ventricles). Time-domain recordings of chamber volume were Fourier-transformed to obtain 
beat rates. The spontaneous beat rate of NRVM ventricles $(\sim 130 \pm 15 \mathrm{bpm})$ was higher than hiPSC-CM ventricles ( $85 \pm 15 \mathrm{bpm}$ ), and both increased by $\sim 40 \%$ following exposure to $10^{-4}$ M Iso. $* \mathrm{P}<0.05, * * \mathrm{P}<0.001$, compared to baseline (no Iso), one-way ANOVA with Tukey post-hoc test. Exact $\mathrm{P}$-values for Iso-dependent beat rates $(\mathrm{Hz})$ were $\mathrm{P}=0.0325$ (NRVM, $\mathrm{N}=8$ ventricles) and $\mathrm{P}=0.524$ (Cor.4U, $\mathrm{N}=4$ ventricles). Exact P-values for Isodependent beat rate ( $\%$ increase) were $\mathrm{P}<0.001$ for baseline NRVM versus the highest Iso dose of $10^{-4} \mathrm{M}$. Exact P-values for NRVM at the smallest three doses $\left(10^{-10} \mathrm{M}, 10^{-9} \mathrm{M}\right.$, and $10^{-8} \mathrm{M}$ Iso) versus the largest dose $\left(10^{-4} \mathrm{M}\right.$ Iso) were $\mathrm{P}=0.003, \mathrm{P}=0.006$, and $\mathrm{P}=0.008$, respectively. The exact $\mathrm{P}$-value for NRVM dosed with $10^{-9} \mathrm{M}$ Iso versus $10^{-4} \mathrm{M}$ Iso was $\mathrm{P}=0.011$. For Cor.4U, differences in beat rate were not statistically significant $(\mathrm{P}=0.183)$. In all cases, measurements were performed on day 14. Data are presented as box plots with individual data points overlaid, where lower or upper edges of the box represent $25^{\text {th }}$ or $75^{\text {th }}$ percentiles, the middle bar is the median, dashed red bar is the mean, and whiskers are minimum and maximum values. 


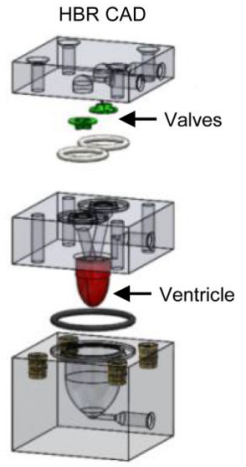

Assembled HBR

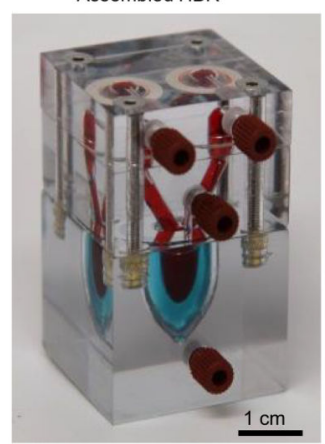

HBR front view

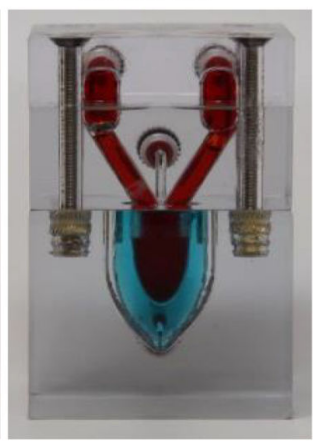

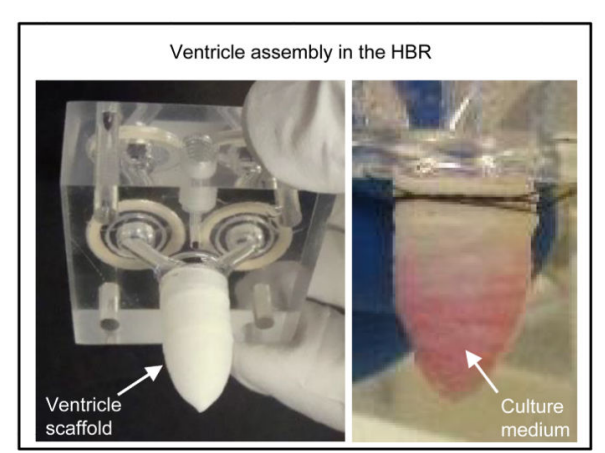

b

C

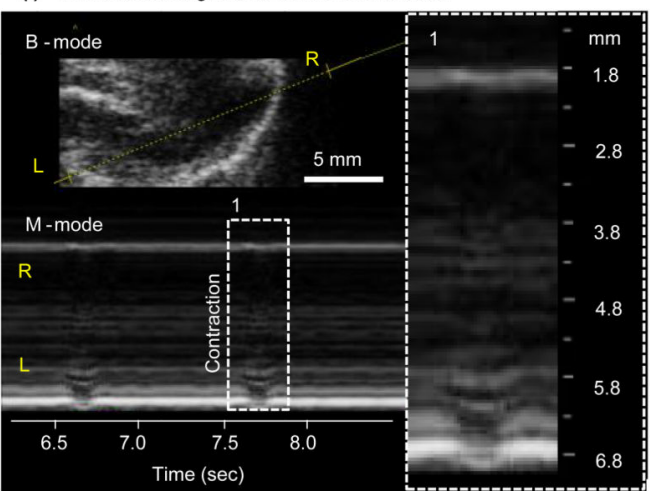

Ventricle catheterization in the HBR

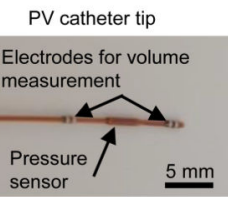

Catheter inserted through HBR port
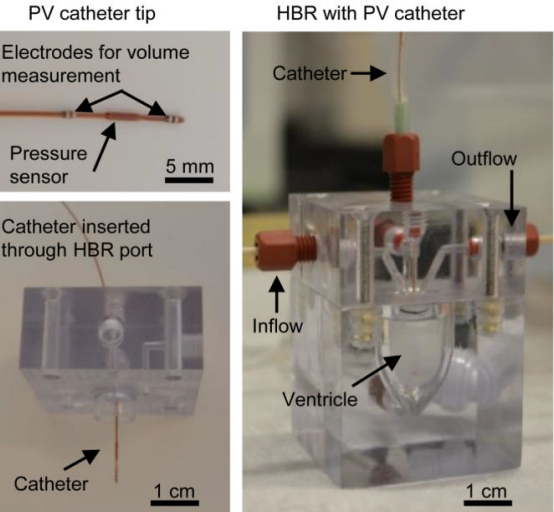
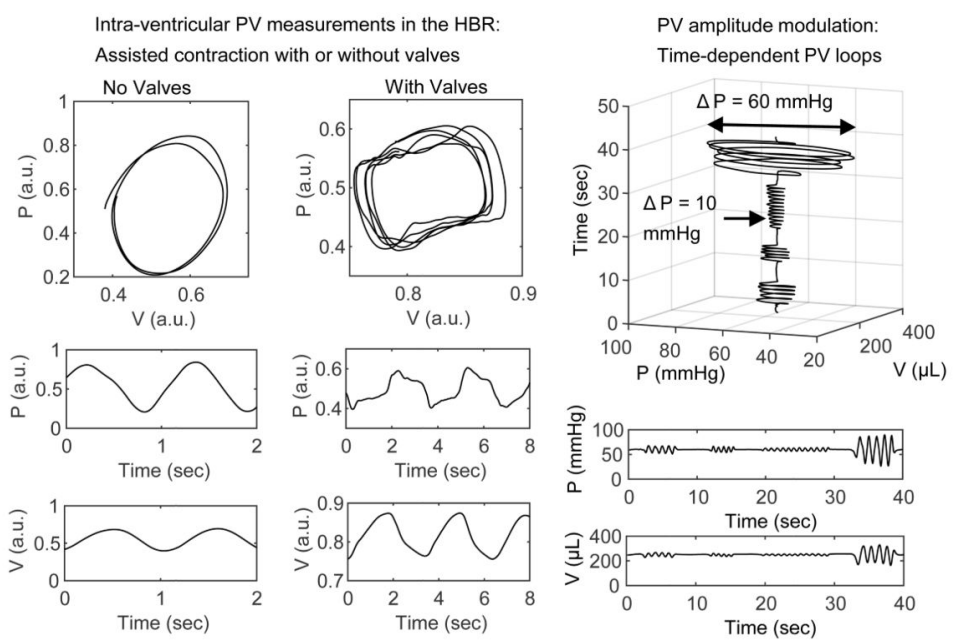

(ii) Ventricle scaffold contraction by HBR assist

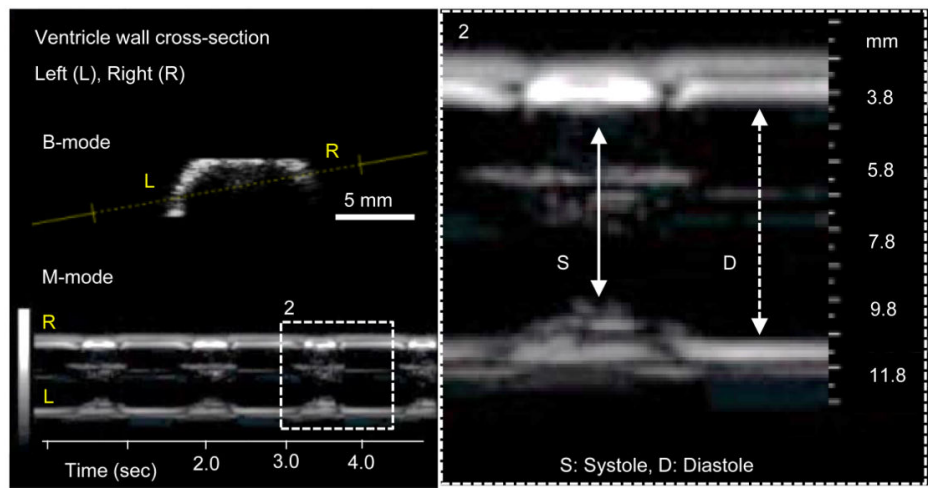

Figure 4|. A heart bioreactor (HBR) for tissue-engineered ventricle culture, assisted contraction, and instrumentation.

a, An overview of ventricle and valve (optional) assembly within the HBR. A computer aided design (CAD) drawing of HBR components shows valve and ventricle placement. The ventricle wall separates intra- and extra- ventricular flow loops, which are indicated by red and blue dyes in the assembled HBR. Ventricle scaffolds are sutured over a support ring where input and output channels of the intra-ventricular flow loop converge. Pressure supplied by an external source to the extra-ventricular flow loop drives assisted ventricle 
contraction and flow through the intra-ventricular flow loop. b, Ventricle catheterization in the HBR enabled pressure and volume measurements during assisted ventricle contraction with or without cast-molded silicone tricuspid valves. c, Echocardiographic measurement of a tissue-engineered neonatal rat ventricular myocyte (NRVM, day 14) ventricle contracting unassisted (left) and a cell-free ventricle scaffold contracting by HBR assist (right). In both cases, cross-sections (brightness mode) and time-dependent traces of the ventricle wall (motion, M-mode) are shown. 


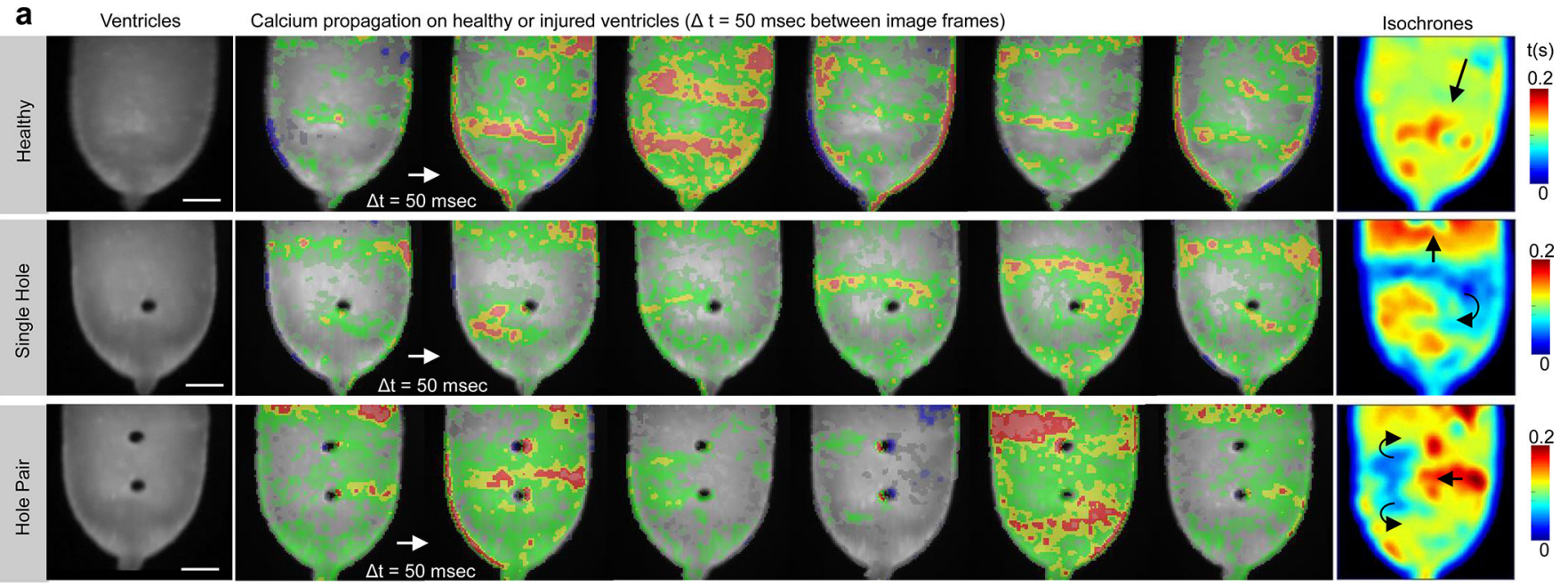

b Rotor in a single hole model injury ( $\Delta \mathrm{t}=40 \mathrm{msec}$ between image frames)

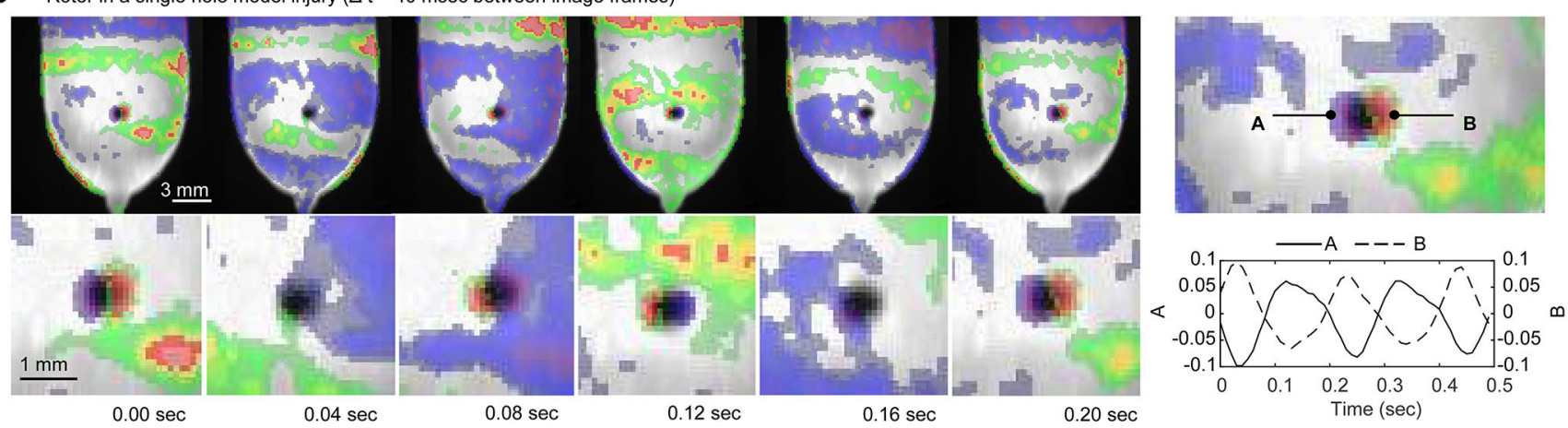

Figure 5|. Structural arrhythmia disease model.

a, Calcium wavefront propagation on a healthy (top) or injured (bottom) tissue engineered neonatal rat ventricular myocyte (NRVM) ventricle. The uninjured ventricle exhibited plane waves with a peak-to-peak spatial period of $\sim 5 \mathrm{~mm}$ (top). Subsequent injury of this ventricle using a $1 \mathrm{~mm}$ diameter biopsy punch resulted in circular anatomical defects that generated pinned spiral waves. The single-hole injury generated a single spiral wave whereas the holepair generated counter-propagating spiral waves that converged and propagated through the inter-hole region with each cycle. $\mathbf{b}$, Calcium fluorescence intensity measurements near the rotor poles of the single hole injury showed consistent phase difference and a rotation rate of $\sim 5 \mathrm{~Hz}$. Experiments were performed at day 12 and spontaneous activity was recorded without external stimulation for all cases. Measurements shown in (a) were acquired using a $5 \mathrm{msec}$ exposure window, whereas those shown in (b) were acquired using a $10 \mathrm{msec}$ exposure window. In all cases, the temporal derivative of calcium fluorescence intensity is displayed as a heat map ranging from blue $(\min )$ to red $(\max )$, overlaid on a greyscale image of the ventricle surface. 